\title{
The neural basis of multitasking
}

Garner, K.G., \& Dux, P.E.D.

Visualisations by K.G. Garner \& David Lloyd.

As humans, we show striking adaptability in our behavioural repertoires. Across the globe, we build shelters and craft attires that are suited to the local conditions. We develop mathematics and technology that allows us to make new connections across societies. We even rapidly engage in large-scale reorganisation of our social conduct to mitigate the threat of a novel virus. Despite this apparent ability to adapt and assimilate a large variety of behaviours to suit our current contexts, we show some striking limitations in how many tasks we can perform at any given moment. Although some behaviours can appear to be performed concurrently with ease, such as talking while walking, we show a stark inability to combine other tasks and skills; imagine compiling a grocery list while recalling your partner's phone number. The observation that attempting to perform multiple tasks concurrently results in at least one of those tasks being performed more slowly, and with less accuracy, was among the first to be made when cognitive psychology was emerging as a scientific discipline (Telford, 1931; Welford, 1959). Interestingly, among these early observations, it was also noted that such performance costs can to some extent be reduced with practice (Telford, 1931), suggesting malleability in how tasks are performed that carries consequences for multitasking operations. Since then, efforts have been made into understanding the nature of the putative operations that give rise to multitasking costs, the neural computations and architectures that instantiate those operations, and the neural and functional changes that drive improvements in multitasking performance. The aim of this chapter is to synthesise the in-roads that have been made into understanding the neural basis of multitasking costs and their practice-induced remediation, and to use that knowledge to propose the next steps forward in our understanding.

\section{Approaches to understanding the neural basis of multitasking}

Approaches to understanding the neural basis of multitasking have largely followed three theoretical traditions. Here, we provide a taxonomy of the theories and empirical insights that have been garnered from these three approaches (see Figure 1). On one hand, researchers have used models of the cognitive architectures that are designed to explain multitasking costs in order to make predictions of what should be observed at the neural level (see Marois \& Ivanoff, 2005). In this approach, which we shall refer to as the cognitive architectures approach, the startpoint is a model that provides a descriptive account or a computational representation of the operations that could underpin multitasking performance. Given the set of procedures or operations posed by a model, researchers then seek to make predictions regarding the neural correlates that should be observed when humans perform tasks designed to capture the cognitive phenomena of interest, in the current case multitasking. As a relevant example; the act of making the correct response given a sensory input, such as waving hello upon 
seeing a friend approaching, is assumed by multiple models to be composed of several distinct processing stages (Brown \& Heathcote, 2008; Ratcliff \& McKoon, 2008; e.g. Sternberg, 1969) such as identification (it is your friend's face), response selection (a wave is better than a frown) and response execution (your hand moves from side to side). If something happened so that completing a stage is more difficult (your friend is being responsible and wearing a covid-mask), then the duration of the relevant stage should be increased. Researchers can translate such anticipated duration changes to what should be observed in recorded brain activity. In this example, neurons that care about response-selection should be active for longer, therefore signals that correspond to neural energy usage, such as the blood-oxygenation dependant signal (BOLD) detected by functional Magnetic Resonance Imaging (MRI), should be stronger when choosing between a wave or a frown when either is more difficult. In the discussion below, we show how such models have generated the hunt for neural correlates in the context of multitasking performance, and how a frontoparietal network has been implicated as important, regardless of the specific motivating model.

A second approach offers an alternate inferential route; going from observations of systems-level neurophysiological phenomena to hypotheses regarding plausible operations that underpin multitasking performance, given the observed neural behaviour of the system. We refer to this as the systems architecture approach. Rather than taking a specific model for multitasking performance, this approach instead asks which observations of neural activity could be relevant to the formation of a hypothesis regarding multitasking operations, given the key behavioural signatures of multitasking performance. For an example of this approach, and as will be discussed in more depth below, the observation that multitasking costs are pervasive across most mental tasks has motivated investigations into which brain systems show sensitivity to task relevant information that is convergent across multiple domains such as attention, memory and response-selection (Duncan, 2010, 2013). Similarly, the observation that multitasking costs reduce with practice suggests that a study of the brain's learning architectures may garner clues for how experience-dependant changes in task representations may give rise to attenuated multitasking costs (Dux et al., 2009; Garner et al., 2020; Garner et al., 2014; Garner \& Dux, 2015). This approach has implicated sensitivity of a common set of frontal, parietal and striatal regions to information pertinent to the current task goals, such as the identity or the colour of the green ' $A$ ' on the screen, dependent on which aspect of the letter you are currently reporting. The question that follows is: what kind of functional properties could these region's constituent neurons carry that would give rise to this observed phenomena and also give rise to multitasking costs, and their practice-related attenuation? We show how the concept of neurons that adapt their responses to encode the current cognitive episode can provide explanations as to why the human brain shows great flexibility in the behaviours that are performed, at the expense of multitasking capability. Specifically, neurons that respond to multiple tasks need to be shared between tasks and the extent of this sharing may give rise to multitasking costs.

The third approach, which we call the neural architecture approach, derives models inspired by what we know about how neurons function and relates their computational properties to how individuals undertake multiple tasks concurrently. In this approach, researchers leverage models that characterise neural function, in order to 
build networks that perform tasks analogous to the cognitive phenomena of interest. For example, functions describing how the membrane potential of a neuron changes in response to synaptic inputs and injected currents (see Dayan \& Abbott, 2001) can serve as computational units within a network. By constructing a simulated task environment within which the network can perform, researchers can determine which assumptions about connectivity rules produce responses that are comparable to observed behavioural or neurophysiological data. Such assumptions include whether a given unit is excitatory or inhibitory, from where units receive inputs or project outputs, and what causes the coupling strength between units to increase or decrease. By making explicit these assumptions, we can make inferences regarding how a system can be wired to effectively produce behaviour, what kinds of computations the system must perform to produce the observed outputs, and which advantages and disadvantages this confers for new tasks or operations. We show below how these approaches have yielded further insight into the advantages of systems that share units between constituent tasks. Neural sharing may drive behavioural flexibility and facilitate learning of novel tasks, at the direct expense of multitasking ability.

Throughout our taxonomy of investigations into the neural basis of multitasking, we seek to consider the ensuing insights in light of what we believe to be key criteria for understanding how neural function gives rise to multitasking costs. According to computational biologist David Marr (1982), understanding of the central nervous system occurs at three levels; first, we can understand the nature of the problem that is expressed - as in, what is the problem that the brain is solving? Here, an initial answer to this question could be 'the problem of performing multiple tasks concurrently and accurately'. However, we will argue that what is revealed from our taxonomy is that this question is surprisingly difficult to answer definitively, and yet how one answers it question has significant implications for how we derive the subsequent stages of understanding. The second level advanced by Marr concerns the algorithmic, or representational level; i.e. how does the system do what it does? How does the system concurrently represent multiple stimuli and goals, and what procedural manipulations are acted upon those representations to produce multiple responses that are timely and accurate? We show how empirical investigations into the neural basis of multitasking have largely sought to understand which putative algorithms are most likely, given assumed mappings between certain assumed procedural features and observable neural correlates. The third level of understanding concerns the physical implementation of such operations ; i.e. how could the brain's neural architecture instantiate the representation and performance of multiple tasks? We discuss some initial insights into this level of understanding the neural basis of multitasking, and suggest avenues for further investigation. 


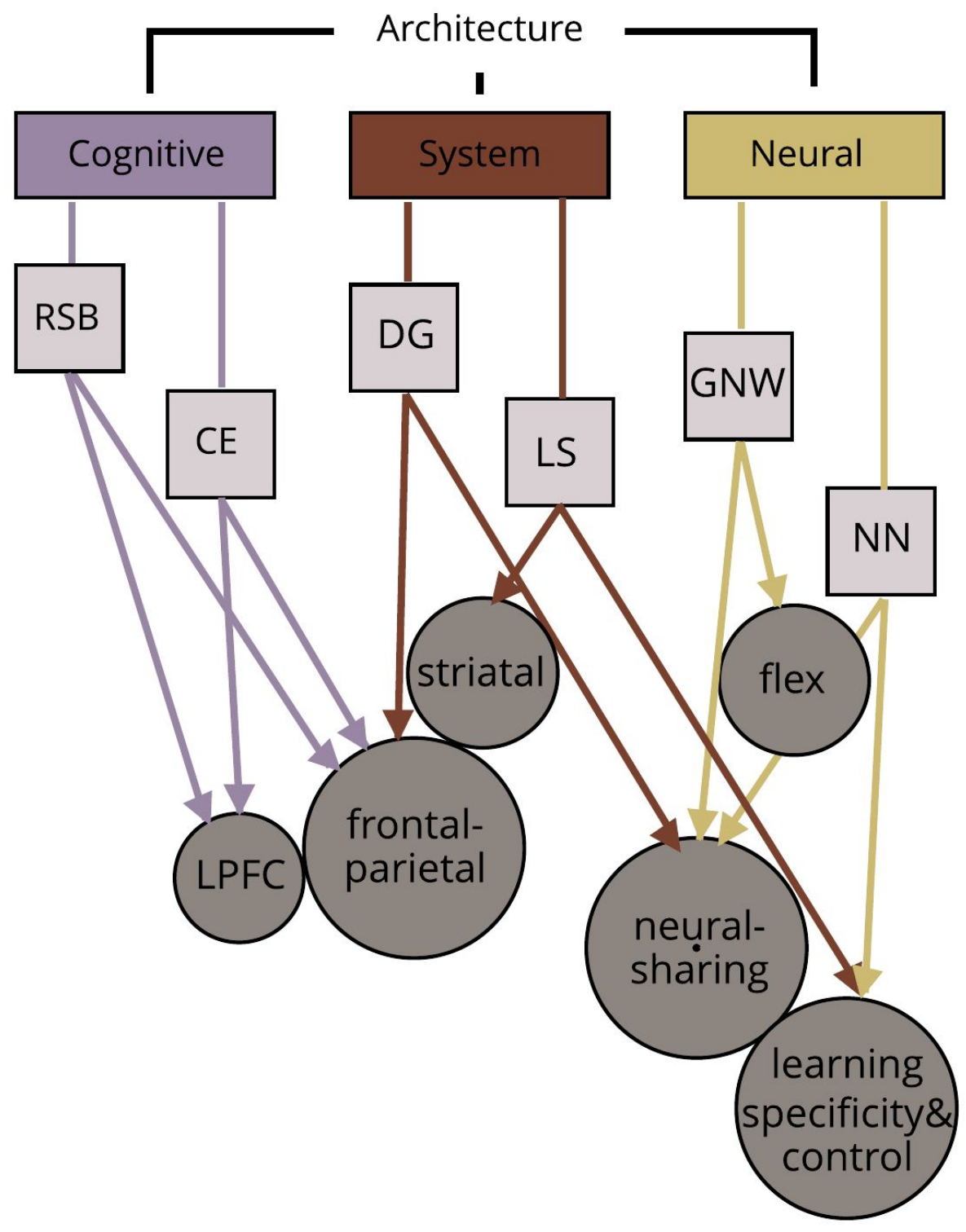


Figure 1: A taxonomy of the approaches towards understanding the neural basis of multitasking. Three broad approaches are identified by the taxonomy; cognitive architectures, system architectures, and neural architectures. Specific theoretical positions (squares) within each approach have yielded convergent and unique conclusions (circles) regarding the neural basis of multitasking. Response-selection bottleneck $(R S B)$ and central executive (CE) models have implicated a set of frontal-parietal regions, and in particular the lateral prefrontal cortex (LPFC) as underpinning multitasking performance. Observations of task performance across multiple domains $(M D)$ have similarly implicated the importance of frontal parietal regions, and have motivated the theory that associative neurons coding relevant information across tasks must be shared between tasks during multitasking, giving rise to costs. Studies of the brain's learning systems (LS) have extended the hypothesis space by suggesting that striatal-cortical connections are part of the neurophysiological infrastructure that gives rise to multitasking costs. Moreover, findings garnered from this approach suggest that multitasking costs may stem from an initial over recruitment of shared neurons, possibly owing to error in encoding, or insufficient experience to differentiate the task space. A spiking neural network model of a global neuronal workspace (GNW) shows that a system containing neurons that are recruited to route task-relevant information across tasks can give rise to flexible behaviour (flex), but requires a serial processing strategy, thereby causing multitasking costs. Neural network $(N N)$ models (defined in text) show that systems consisting of units that contribute to multiple task representations (and are thus shared between tasks) are better able to generalise previous learning to novel tasks, at the expense of multitasking capability.

\section{Observing the multitasking brain}

Although we shall argue throughout this chapter that it is a challenge to pin down exactly what problem the brain is solving when we observe incurred multitasking costs (i.e. the 'why' of multitasking costs), we can certainly characterize what we do observe when participants multitask in the lab. Multitasking has been investigated with an array of paradigms, that have combined everything from unspeeded perceptual judgements (Raymond et al., 1992), to continuous complex tasks such as prose comprehension (Cho et al., 2015) and driving (Levy \& Pashler, 2008). Here we largely focus on paradigms requiring the speeded completion of two temporally overlapping sensorimotor tasks, as this is where the majority of efforts to understand the cognitive architectures underpinning multitasking have been focused (Logan \& Gordon, 2001; Meyer \& Kieras, 1997; Navon \& Miller, 2002; Pashler, 1994; Salvucci \& Taatgen, 2008; Tombu \& Jolicœur, 2003). Constituent tasks in these paradigms typically involve mapping two or more stimuli, such as letters or sounds, to the appropriate mototric output, such as a key press or a vocal response.

In many multitasking paradigms, task 2 is presented subsequent to task 1 , at increasing intervals, typically ranging from $100 \mathrm{~ms}$ to $1000 \mathrm{~ms}$, in order to vary the demands placed on the system (see Pashler, 1994 for a review). Under these conditions, it has been consistently observed that the duration of task 2 performance 
increases as the intervals between the two tasks decreases. Slowing to task 1 performance has also been observed, more variably, but particularly when the two tasks are presented simultaneously (Maquestiaux et al., 2008; Welford, 1959). Importantly, multitasking costs are evident even when tasks do not overlap in the sensory modality of stimulus inputs and response outputs (Hazeltine et al., 2006; Hazeltine \& Ruthruff, 2006; Pashler. 1990). For example, one task can entail making a manual response to a visual input while the second requires a vocal response to an auditory output - even under such conditions substantial multitasking costs are found. This suggests that the locus of multitasking limitations lay outside the (initial) parsing of sensory inputs (Johnston \& McCann, 2006), or the execution of a motor-response (Klapp et al., 2019). However, the mapping between modalities can influence multitasking performance, for example, costs are lower when pairing visual-manual with auditory-vocal tasks relative to when pairing auditory-manual and visual-vocal tasks (Hazeltine et al., 2006). The reason as to why certain modality pairings confer performance benefits remain to be fully elucidated, but may relate to the frequency with which modalities are naturally paired. For example, we gain experience producing vocalisations in response to auditory inputs with each conversation we conduct.

Observations that task 1 performance was largely spared from multitasking costs (Pashler, 1984) motivated 'bottleneck' theories which proposed that task 2 slowing was caused by processing limitations of a single channel at the locus of task 1 response selection; ie. while task 1 processing proceeded unimpinged, task 2 response selection had to await for availability of the serial processing mechanism (Pashler, 1994). Moreover, the finding that practice on task 1 alone is sufficient to reduce or even overcome multitasking costs (Ruthruff, Hazeltine, et al., 2006; Ruthruff, Van Selst, et al., 2006; Van Selst et al., 2003), suggests that reducing the amount of time required for the execution of task 1 operations causes reduced impingement on task 2 operations, which is in accordance with the assumptions of the bottleneck model. However, the single-channel serial bottleneck model is an unlikely candidate to account for the consistent observations that task 1 performance is affected in many multitasking contexts (see Strobach et al., 2015 for a review), that the response selected for task 1 can be influenced by the response required for task 2 (Bratzke \& Janczyk, 2020; Janczyk et al., 2018; Ko \& Miller, 2014; Miller \& Alderton, 2006), and that sensory evidence can be accumulated towards the task 2 decision during performance of task 1 (Zylberberg et al., 2010). If multitasking costs are to be thought of as stemming from limitations in a mechanism that supports response-selection, as has occurred in some investigations into the neural correlates of multitasking, then the data largely favour modified accounts of the bottleneck model that postulate that multitasking costs are due to limitations of a mechanism that supports response-selection and allocates this capacity between the two tasks (Tombu \& Jolicœur, 2003). It is also worth noting that the conclusion that the limitations that give rise to multitasking costs are located at a central response-selection stage are by no-means conclusive. For example, theory and data have supported the idea that the bottleneck that gives rise to multitasking costs may be motoric in nature (Bratzke et al., 2009), and that a bottleneck exists at the point of timing the initiation of a pre-programmed response (Klapp et al., 2019), which is something that can presumably only occur once a response has been selected. However, we have largely focused on 'central bottleneck' 
accounts here, as this account has driven a substantial portion of the cognitive architecture efforts to understand the neural basis of multitasking.

The above mentioned bottleneck theories postulate that multitasking costs are observed because of delays incurred to the performance of the task itself. It is also possible that multitasking costs arise due to functions the brain must perform as a consequence of being required to manage multiple tasks. To wit, the whole of multitasking is greater than the sum of the constituent tasks. For example, an executive system may be required to programme and schedule task performances when multiple tasks are present (Logan \& Gordon, 2001; Meyer \& Kieras, 1997; Salvucci \& Taatgen, 2008). Investigators have sought to identify such extra sources of slowing that may contribute to multitasking costs. For example, completing single tasks in a mixed block of trials, where the participant does not know ahead of each trial which of the two single tasks will be presented, results in longer response times relative to when a block only contains one single task type (Pashler et al., 2000). This suggests that establishing the task set, i.e. the implemented rules for mapping a given sensory input to the task goal, may be one source of slowing that causes performance costs under multitasking conditions. Moreover, the observation that practice on constituent tasks that are presented together as a multitask results in larger reductions in costs than when both the tasks are practiced as single tasks (Strobach, 2019), suggests that something beyond the execution of each task can be trained to influence performance. Such findings have been interpreted as support for theories postulating that a central executive system is recruited to manage the execution of multiple tasks.

Collectively, these empirical features have helped inform the models derived to explain how multitasking costs arise, and have therefore shaped the landscape of insights yielded by the first body of work in our taxonomy; i.e. the investigations seeking to determine whether neural responses exhibit features that would be expected given the existence of a response-selection bottleneck or a central executive that gives rise to multitasking limitations.

\section{Cognitive architectures: Hunting the putative neural correlates of a response-selection bottleneck or a central executive}

In order to seek evidence for which cognitive architectures could likely give rise to multitasking costs in the human brain, researchers have sought to extrapolate how a proposed cognitive mechanism could be manifest in a neural correlates. Empirical investigations have then been constructed to determine whether or not the evidence suggests the presence of such a correlate. This is an immensely difficult task given that these models are not couched in the functions of neurons, and that the neuroscientific tools available to researchers when undertaking such work with human subjects result in observations of neurobiological function that are indirect. For example, functional Magnetic Resonance Imaging, which has often been applied in this context (Marois \& Ivanoff, 2005), measures the movement of oxygen concentration in the blood (blood-oxygenation dependent signal; BOLD), which serves as a proxy for the energy usage incurred by increased neural activity (Logothetis, 2008). Moreover, the spatial resolution of MRI is quite large $\left(\sim 1-3 \mathrm{~mm}^{3}\right)$, with each voxel consisting of hundreds of thousands of 
neurons ${ }^{1}$, therefore each voxel likely contains a mixture of underlying neural responses on any given observation. As such, any interpretations of such studies must include the caveat that we currently do not have a forward model that can map for us exactly what we should see in the observed neural signal, given the proposed cognitive operations, and that multiple other explanations for the observed neural correlates likely remain viable.

\section{Response-selection bottlenecks}

Bottleneck models of multitasking limitations inherit their structure from the concept that the performance of a task can be parsed into processing stages (Sternberg, 1969). In this case, sensorimotor performance is assumed to be composed of three distinct stages; perceptual and response execution stages that encompass the analysis of incoming sensory inputs and the process of physically performing the movement required by the manual response, and a central stage that maps the input to the appropriate output. Of import, it is this central stage that is hypothesised to be limited in processing capacity. According to theoretical accounts which posit that the locus of multitasking costs lay at the central stage of sensorimotor processing (Pashler, 1994; Welford, 1952. 1967), performance slowing occurs when the interval between two tasks is short, as task 2 central stages must wait for sufficient availability of the underlying mechanism. Consequently these stages may not proceed until task 1 central stages are complete. The strength of this theory lay in its simplicity and the quantitative predictions for behaviour that have largely been corroborated (Pashler, 1994; Tombu \& Jolicœur, 2003), although there have been many observations of phenomena not captured by these models (Huestegge \& Koch, 2010; Ko \& Miller, 2014; Miller \& Alderton, 2006; Navon \& Miller, 2002; Ulrich \& Miller, 2008; Zylberberg et al., 2010). Nevertheless, predictions made by these models regarding the duration of central processing stages have motivated searches for the neural correlates of this response-selection bottleneck. For example, a key prediction made by these models is that task 1 difficulty manipulations should delay the start and end times for which task 2 response selection occurs. This has motivated the hypothesis that any brain regions supporting such a mechanism should show activity (i.e. changes to the BOLD response) that scales with task 1 difficulty manipulations. Importantly, any such brain region should also show sensitivity when each single task is performed in isolation, given that the proposed central stage of processing is assumed to underpin performance of each sensorimotor task. A series of studies have indeed demonstrated BOLD activity changes that fit such a profile, and that this pattern is typically and consistently observed in the left lateral prefrontal cortex (LPFC). For example, Marois et al (Marois et al., 2006) manipulated the number of choice alternatives for tasks administered under both single-task and dual-task conditions, and observed increased peak BOLD amplitude in the dorsal pre-motor and lateral prefrontal cortices. These regions were also insensitive to a perceptual difficulty manipulation that increased task 2 performance time without influencing response-selection demands; one interpretation of this finding is that these regions specifically reflect the response-selection limitations that are assumed by the central bottleneck model.

One issue with using $\mathrm{fMRI}$ to interrogate the neural correlates of response-selection limitations (or, cognition in general) is that the temporal resolution of the signal is poor. A typical scan of the human brain takes

\footnotetext{
${ }^{1}$ https://cfn.upenn.edu/aguirre/wiki/public:neurons_in_a_voxel
} 
around 1.5-2 seconds, which is longer than that required to perform the sensorimotor tasks employed in multitasking paradigms. However, it is possible to adjust experimental manipulations and/or analysis techniques in order to match response-selection demands to the temporal resolution of MRI. For example, Dux et al. (Dux et al., 2006) sampled brain activity while participants completed single and multitask conditions with sufficiently taxing response demands to bring RTs within the temporal resolution of fMRI. This approach carries the assumption that there is a quantitative, rather than qualitative change, in how the task is performed under easy relative to hard conditions. The authors then leveraged the central bottleneck model to derive predictions about the shape of the hemodynamic response function (HRF) that should be observed in brain areas that underpinned such a bottleneck. The HRF characterises the rise and fall of the BOLD signal in response to experimental perturbations. The prediction was that the duration of the HRF should be extended in multitask, relative to single-task conditions, given that a brain region underpinning response selection limitations would contribute computations for a longer period in the multitask condition. Activity in LPFC showed a protracted response signature that corresponded to the increased response-selection demands, corroborating Marois et al. (Marois et al., 2006) to show that activity in this region matches the anticipated signature of a central, response-selection limitation that gives rise to multitasking limits. Moreover, this group of researchers showed that this protraction of LPFC activity was reduced after participants practiced the multitask over multiple sessions (and therefore reduced multitasking costs) (Dux et al., 2009), which is in line with the prediction that practice reduces the duration of response-selection for task 1. However, this practice study did not contain a control group, so it is hard to disentangle whether it was a practice-induced attenuation of multitasking, or some other factor such as reduced task novelty, or motivational factors, that drove the result.

The above studies illustrate that it is possible to find a putative neural correlate of central capacity limits in the human LPFC. As will be seen in the next section, this brain region consistently shows activity patterns that can be inferred as corresponding to the increased cognitive demands induced by attempting multitasking. This is comforting in the respect that it appears that this region consistently shows sensitivity to multitasking demands. In the current context, the observed patterns of activity have been taken as showing the viability of models that propose a central limitation that struggles to support multiple response-selection demands. In the next section, we shall see how a comparable approach seeking to find correlates of a central executive that schedules the execution of multiple tasks also elicits findings that converge on the LPFC as a region of importance (see Figure 2). 

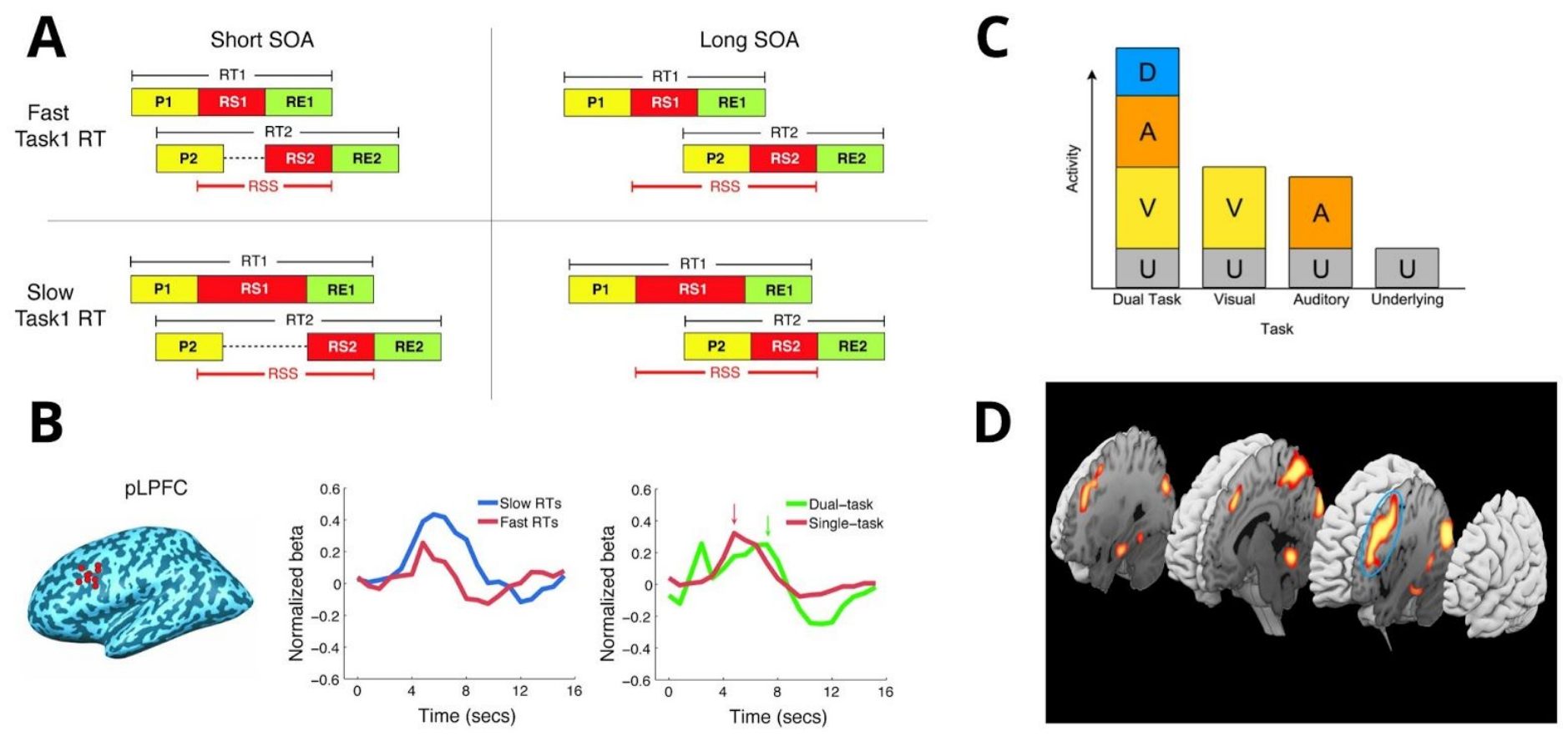

Figure 2: Showing the logic and most consistent findings from investigations seeking to map either the response selection bottleneck or the central executive to observable neural correlates. Panel A) The central bottleneck model with Perceptual (P), Response Selection (RS) and Response Execution (RE) stages of sensorimotor processing (Pashler, 1994). According to the model, the duration of engagement of central and limited response selection stage (RSS) is extended for slow response times and for dual relative to single-task conditions. If a brain region contains neurons performing such a response-selection function, it is assumed that the haemodynamic response function (HRF) will show a protracted response for slow relative to fast response times (RTs), and for dual relative to single task trials. B) Such a response has been most consistently observed in the posterior left lateral prefrontal cortex (pLPFC). C) The logic of investigations seeking to map neural correlates to a 'central executive'. As something extra is recruited to manage the performance of multiple tasks, brain regions containing neurons underpinning such an operation should show a response in this condition that is greater than the sum of the visual (V) and auditory (A) single tasks that make up the multitasking operation. D) This approach has yielded a response pattern that consistently includes the pLPFC (circled in blue). Panels A \& B are taken from (Dux et al., 2006), panel C is reproduced from (Szameitat et al., 2011) \& D is reproduced from (Schubert \& Szameitat, 2003).

Seeking the central executive

Just as the response-selection bottleneck model has been used to derive hypotheses about what one could expect to observe in fMRI signals, so too has the concept of a 'central executive' motivated a comparable body of work. Importantly, theories that postulate that multitasking costs are due to an overarching executive process that monitors and manages task schedules do not assume that there is a capacity limitation in the function of mapping a stimulus input to a response output. Multitasking costs have instead been attributed to stemming from the following; an adaptive task scheduling mechanism that acts to reduce overload of peripheral processors (Meyer \& Kieras, 1997; Meyer \& Kieras, 1997), a serial processor that coordinates subtasks to reduce resource conflict (Salvucci \& Taatgen, 2008), and an executive module that adjusts the parameters for evidence accumulation, given the current task set (Logan \& Gordon, 2001). Across all these models, there is the notion that 
multitasking involves a set of cognitive functions that are greater than the sum of the constituent task parts; i.e. something extra is required in the multitasking context that is not required in the single task context. Thus, the approach typically taken in studies looking for a putative neural correlate is to find brain regions that show greater BOLD activity than is observed by the summed activity elicited by component tasks. Moreover, and in contrast to the central bottleneck model, such brain regions need not necessarily overlap with brain regions that are recruited to perform single sensorimotor tasks.

Investigations utilising such a method have uncovered neural correlates spanning the lateral prefrontal cortex (Collette et al., 2005; Schubert \& Szameitat, 2003; Szameitat et al., 2002, 2006), middle frontal gyrus (Szameitat et al., 2002), anterior cingulate cortex (Dreher \& Grafman, 2003), parietal cortex (Collette et al., 2005; Schubert \& Szameitat, 2003; Szameitat et al., 2002), and subcortical regions (Collette et al., 2005). However, once task-preparation processes have been controlled for, regions showing greater activation in dual-task relative to single-task conditions broadly overlap with the regions elicited by the single tasks alone (Erickson et al., 2005). In a potential challenge to the predictions made by central executive models, at least two investigations have failed to find brain regions that show increased activity specific to the dual-task condition (Dux et al., 2006; Jiang et al., 2004). However, given the poor temporal resolution of $\mathrm{fMRI}$, it is challenging to rule out whether this increased activity was too short to detect. Using a measure of how much BOLD activity in 'sensory processing regions' (for example, the fusiform face area) can be explained by activity in the LPFC under varying task contexts (i.e. single vs dual-tasks), Stelzel et al (Stelzel et al., 2006) showed that activity in task 1 relevant sensory regions was better explained by LPFC activity in the presence of the task 2 stimulus, regardless of whether or not the participant was required to perform task 2 . This has been interpreted as evidence that a protective mechanism is invoked to protect task 1 , that is separate to task 2 performance, as could be expected from a central executive. Furthermore, parametric manipulations of other task conditions that should influence the function of a central executive, such as the predictability of task order, have consistently been associated with increased BOLD activity in the LPFC (Stelzel et al., 2008; Szameitat et al., 2002, 2006).

It is interesting that regardless of the theoretical motivation, that BOLD activity in the LPFC has covaried with experimental manipulations of multitasking demands. However, it is difficult to discern exactly what these BOLD activity changes reflect in terms of putative algorithms, given the lack of specificity in the forward mapping from proposed cognitive operations to observed neural responses. What these results do show is that any effort to determine how the neurophysiological hardware could support the putative mechanisms of multitasking should take into account the nature of inputs, outputs and local LPFC processes, and perhaps leverage this information to narrow down exactly how a biologically plausible model would act to give rise to multitasking costs. Furthermore, some clues to the cause of this convergence may potentially be found in the rodent and monkey dual-tasking literature, where neurophysiological recordings suggest that there is indeed a network that fits the proposed profile of a central executive, and that it is intertwined with the circuits that support single-task processing in the prefrontal cortex (Lebedev et al., 2004; Messinger et al., 2009; Olton et al., 1988; Pang et al., 2001). 
At this point, we understand that performance suffers when we seek to undertake two tasks. The models and/or algorithms that have been proposed to account for these phenomena include a serial or capacity limited channel underpinning the translation of stimulus-inputs to response-outputs, and/or an executive mechanism that titrates task parameters or schedules the performance of sub-tasks in accordance with available resources. In all cases, the search for proposed neural correlates have largely converged on predicted patterns of BOLD activity being most prominently observed in LPFC. It is interesting to consider how these strands of inquiry inform the 'why' of multitasking; specifically, what problem is the brain solving when we observe multitasking costs? Bottleneck models focus solely on the successful performance of two sensorimotor tasks. It is indeed true that we are often required to perform multiple tasks, but a deeper consideration of the 'why' question does suggest that bottleneck models (in this case) largely serve to predict the empirical phenomena of multitasking costs, rather than demonstrating why a system would organise in a manner that induces a single or limited capacity-channel for response-selection. On the other hand, models proposing a central executive do probe further into the 'why' question; these models suggest that the performance of multiple tasks requires resource management, and that multitasking management often (and optimally) involves delaying access to resources for certain subtasks. Largely though, these models assume that the problem the brain is seeking to solve is the performance of multiple tasks; i.e. the brain is trying to solve multitasking. Later in this chapter, we will see that models that seek to emulate the function of neurons in systems that can perform sensorimotor tasks have yielded some rather different answers to this question.

\section{System architectures: Using neurophysiological observations from broader domains to shed insight into the neural basis of multitasking}

Of course, our understanding of the neural basis of multitasking need not only be informed by experimental manipulations of human multitasking scenarios and the models that seek to explain the observed phenomena. Interesting insights have been leveraged from the broader literature on domain general human cognition (Duncan, 2010, 2013) and animal models of sensorimotor action (Watanabe \& Funahashi, 2018). In the case of the former; by observing the patterns of BOLD responses across a range of complex cognitive domains such as working memory, response-conflict and challenging sensorimotor processing, Duncan $(2010,2013)$ demonstrated that a common set of overlapping frontal and parietal brain regions showed activity covarying with the difficulty of the task, regardless of the specific domain of the paradigm. The inference that followed was that frontal and parietal brain regions constitute a multiple demand network, whose constituent neurons flexibly reconfigure their response properties to be tuned for the current cognitive episode. Duncan (2013) proposed that just as artificial intelligence models can solve complex tasks by breaking them down into more readily solvable sub-goals (Sacerdoti, 1974), so too does the brain, and that the contents of the current mental episode or subgoal are encoded into the frontal-parietal multiple demand system. 
Empirical efforts to determine whether frontal and parietal neurons do exhibit such properties have typically leveraged information-based approaches to the analysis of neural data, to determine which task rule is currently active in a given experimental context (Jackson et al., 2017; Jackson \& Woolgar, 2018; Marti et al., 2015; Tamber-Rosenau et al., 2013; Woolgar et al., 2011, 2016). The research reviewed in this chapter up to this point have largely used activation-based approaches, where the goal is to determine the extent to which the amplitude of the BOLD response systematically changes as a function of manipulations of interest. Given that there are hundreds of thousands of voxels in one brain scan, it is very easy to identify statistically significant voxels by chance. Therefore, under this approach, it is important to identify clusters of voxels that show a comparable association between response amplitude and the experimental manipulations (Nichols, 2012). Thus neighbouring voxels are pooled to answer the questions; 'does this set of voxels (area $\mathrm{x}$ ) show systematic activity changes that correspond to my experimental manipulation?', and 'at a cluster level, do I have more significant clusters than I could expect by chance?' This approach works well to identify whether or not a brain region is responsive to an experimental condition, but provides little information for how well that activation maps to the cognitive operations that may underpin performance in a given experimental condition. For example, although activation may reliably increase between single and multitasking conditions, it does not necessarily follow that the activation change corresponds to a neural code that contributes to multitasking performance. It could, for example, reflect an increasing quantity of stimulus energy on multitask trials.

In contrast, information-based approaches ask 'given this set of voxels (i.e. the voxels in area $x$ ), can I find patterns distributed across the voxels that reliably map to my experimental conditions?'. In effect, this means that a classification algorithm, such as a support vector machine, is trained on a set of voxels to map a function between feature space (i.e. activation level of each voxel) to the experiment space (e.g. experimental conditions, such as specific stimulus-response mappings, or the current task-rule). This classification approach can detect differences between conditions, given a true bias exists in the mapping of neural response to the dimensions of measurement. For example, such classification of $\mathrm{FMRI}$ data assumes a true spatial bias in the voxels that increase their activity given one condition, relative to another (Haxby, 2012; Serences \& Saproo, 2012). Once trained, the algorithm is then tested on new, as yet unseen, data. If classification performance is above chance, then the set of voxels (or timepoints, or whichever features of the data that are under interrogation) are said to contain information about the experimental conditions. Well-controlled experiments can ensure that the only possible difference between conditions is the phenomena of interest - for example, stimuli and responses can be held constant, but the rule that maps them to each other can vary across conditions, increasing the chance that the classification analysis has detected a code in the neural data that pertains to the mapping of stimuli to responses. Given that information-based approaches seek to identify distributed patterns in the data, this approach is also particularly useful when there is reason to believe that the aggregate average BOLD response within a region of interest may increase for, but not differ between two conditions; for example, when the same frontal or parietal brain regions show increased activity in the context of two comparable challenging task rules. 
Applications of this information-based approach has indeed shown that neural activity recorded from the frontal-parietal cortex carries information about which rule maps a stimulus to a response, even when the stimuli and responses themselves are held constant across all conditions (Woolgar et al., 2011, 2016). Moreover, frontal and parietal neural responses in human and non-human primates show activity patterns that adapt to reflect the current task-rule, under experimental conditions where the rules change over the course of a single trial (J. Jackson et al., 2017; Meyers et al., 2008; Stokes et al., 2013; Watanabe \& Funahashi, 2014, 2018). Interestingly, and in support of what would be expected by limited capacity models, such decoding methods applied over neural data with higher temporal resolution than fMRI (e.g. magnetoencephalography, MEG) have shown that frontal and parietal responses show seriality (i.e. one task rule can be decoded at a time), whereas visual and motor responses do not (Marti et al., 2015). Moreover, and in support of the human fMRI work presented above, it has been shown that the activity of LPFC neurons, recorded in awake macaques that have been trained to perform a multitasking paradigm, show a decreased ability to represent task information when the concurrent task demands increase, and that this decrease is proportional to the observed behavioural multitasking costs (Watanabe \& Funahashi, 2014). Given that the same frontal and parietal neurons are assumed to reconfigure their response profiles to encode the current task demands, the interpretation is that multitasking costs may stem from at least two sources; first, each task will comprise multiple subgoals, some of which will be consecutively represented in order to solve the overall problem of completing the multitask. Second, given the same neurons may be recruited to represent each task, the constituent tasks may compete for representation within the multiple demand system i.e. neurons that contribute representations to both tasks are overloaded by the current task demands. Note, we will return to the concept of shared representations in the discussion on neural network models below.

Inspired by this account of multitasking limitations, we sought to understand whether practice induced multitasking improvements could be accounted for by a reduction in shared neural overlap in the multiple-demand system (Garner \& Dux, 2015). Specifically we asked; if tasks compete for representation in the multiple demand network, can a reduction in this competition account for multitasking improvements? To address this question, we recruited 100 participants, in what we believe to be the largest fMRI study into the neural correlates of multitasking conducted to date. All participants completed a simple multitasking paradigm in the MRI scanner, which consisted of performing two sensorimotor tasks, either as single tasks or together as a multitask. During the subsequent week, half the participants practiced performing the multitasking paradigm (over thousands of trials), whereas the control group practiced upon a comparably challenging visual-search task. We chose a visual-search task as we did not expect practice of it to lead to multitasking improvements, and it allowed us to match extraneous factors such as motivation and reward history between our practice and control groups. Interestingly, we found that not only did frontal and parietal brain regions show practice-induced differences that were specific to the multitasking practice regimen (specifically the pre-supplementary area and the intra-parietal sulcus), but so too did the striatum, or more specifically, the putamen. This is in concert with recent calls that the multiple demand system should be extended to incorporate the human striatum (Camilleri et al., 2018). 
To address our specific question, we tested our ability to classify patterns of brain activity in this set of frontal-parietal and striatal brain regions, according to which single task participants were performing, before and after practice. The underlying assumption is that the extent to which the tasks can be classified, using the BOLD response across voxels, should increase as neural overlap in the underlying task representation decreases, i.e. decreased sharing of representations should correspond to an increase in the heterogeneity of the BOLD response across voxels. We then asked whether the extent to which classification improved from pre- to post-practice could predict individual improvements in multitasking practice. We found that this was indeed the case; the greater the increase in classification accuracy from pre- to post-practice, the more multitasking improved (see Figure 3). Furthermore, this finding was specific to the above identified brain regions, and no other brain regions, and to the practice group only. We took this finding as inferring support for the notion that practice improves multitasking by reducing competition for representation within the multiple demand network.

As our findings also implicated that the striatum showed comparable activity changes to frontal and parietal brain regions, we next sought to consider how this neurophysiological structure could possibly interface with the cortex to support multitasking behaviour. Interestingly, striatal-cortical interactions have been implicated in many of the components that make up the process of performing multitasking, but the consideration of their contribution to multitasking performance has been far from extensive (Klapp et al., 2019; Thoma et al., 2008; Yildiz \& Beste, 2015). For example, multiple theoretical and computational models implicate striatal-cortical circuits as the hardware that implement multiple algorithms via which the brain may implement a single sensorimotor decision, such as the arbitration between potential task-related signals (Gurney et al., 2001), sequential sampling of evidence towards possible outcomes (Caballero et al., 2018), or instantiation of task-set rules (Bornstein \& Daw, 2011; Joel et al., 2002). Moreover, it has been theorised that the striatum is well placed to send dopaminergic "teaching signals" to the cortex on the basis of current information, which, owing to the cortex's relatively slower dopamine response dynamics, means that the cortex will only retain task representations that prove to be useful over the longer term (Ashby et al., 2010; Hélie et al., 2015). This is in keeping with theories, developed on the basis of observations in rodents and non-human primates, that the striatum constitutes the neurophysiological architecture that manages the transition between the systems that support novel and practiced behaviours (Graybiel \& Grafton, 2015; Jahanshahi et al., 2015; Smith \& Graybiel, 2013; Yin \& Knowlton, 2006), i.e. between the stages of learning where multitasking becomes more or less difficult. Thus, it appears that the nature of the interface between striatum and cortex changes when tasks are practiced, and this change could mediate the extent of multitasking costs.

We sought to test whether our data could support the notion that the neurophysiology that supports multitasking encompasses the striatal-cortical interface (Garner et al., 2020). To do this, we applied dynamic causal modelling (Friston et al., 2003) to our fMRI dataset. This technique allows one to propose and compare between plausible networks that may underpin the observed data. Specifically, DCM tests how well activity observed in one brain region can be explained by the activity of one or more other regions. In our case, we sought to understand whether our data, recorded during multitasking and after its practice induced remediation, were best 
explained by a network where the cortical nodes (pre-SMA, IPS) influenced the activity of the striatum (putamen), or vice versa, or whether connectivity changes were reciprocal between striatum and cortex. Although we observed some differences between hemispheres (see also Filmer et al., 2013; Filmer, Varghese, et al., 2017), the data were largely best explained by models that allowed the putamen to exert greater influence on the cortical nodes, and where putamic influence on pre-SMA activity was modulated with practice (Figure 3C). We interpreted this result as suggesting that during multitasking, performance decrements are due, at least in part, to a limit in the rate at which the putamen can excite appropriate cortical stimulus-response representations and that multitasking limits may be remediated by changes in the rate of information transfer between the putamen and the pre-SMA. Therefore our data do suggest, in accordance with theoretical models of sensorimotor decision making, that the interface between the putamen and key cortical nodes are a putative neurophysiological substrate of multitasking limitations. It must be noted, however, that DCM does not test whether modelled connections are direct, or mediated by a node not included in the network. Therefore, these results have to be interpreted in light of that caveat.
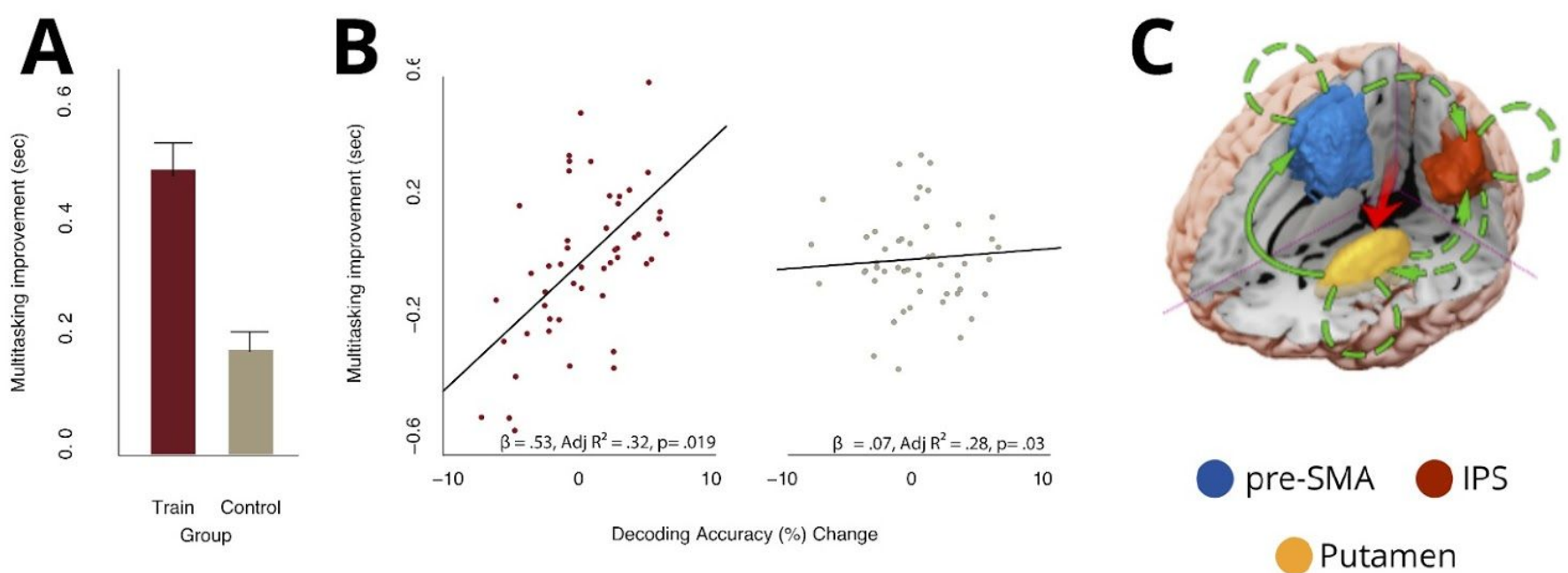

Figure 3: Practice-related improvements in multitasking occur concomitantly with decreased overlap in task representations, and modulations of the putamen's influence on the pre-SMA. A) In our study of 100 participants (Garner \& Dux, 2015), multitasking improvement (y-axis) was larger for the practice (train) group than the control group. B) the increase in decoding accuracy from pre- to post- practice (x-axis) in the brain regions of interest (C) co-varied with performance improvement to a greater extent in the practice group (left panel, red dots) than the control group (right panel, grey dots). C) The brain regions of interest and the change in network dynamics with practice. Coupling between the putamen and the pre-SMA reduced with practice (Garner et al., 2020).

These differing approaches to studying the neurophysiological substrates that underpin multitasking costs offer a subtly yet importantly different answer to the question of why a complex system like the brain may evolve to be such a poor multitasker. Whereas the cognitive architectures approach reviewed above posit that multitasking limits arise from a structural limitation in the system, the concepts arising from the study of the multiple demand and animal sensorimotor systems are instead focused on how a system could operate to 
optimally learn and solve complex problems. The insight revealed by the systems architecture approach is that serial completion of successive subgoals in frontal-parietal-subcortical circuits is a candidate effective method, as is sharing representations in this system between tasks in newer task contexts (see more on this in the discussion below). To wit, these strategies ensure a greater chance of successful performance in complex task spaces. Note that the concept of successive subgoal representation does not necessarily explain multitasking costs per se, as it does not explain why two chains of successive tasks could not be completed at once. On this point, learning and reinforcement models (Sutton \& Barto, 1998) can potentially provide further insights. For example, when the goal of the agent is to learn by reinforcement, it is important to not over-represent any task with extraneous information from other tasks. This ensures that rewards only reinforce the exact representations that caused the attainment of reward, and prevents the agent over-associating non-causal behaviours to reward gain. Under a purely parallel system, an organism may well attribute reward to all ongoing behaviours and therefore engage in an array of superstitious rituals in order to attain a simple cookie. We return to the concept of task overrepresentation, and how practice may act to reduce it and thereby alleviate multitasking costs, in the final section of this chapter.

\section{Neural architectures: how models of neural functioning shed insights into multitasking systems.}

All of the approaches mentioned above have taken observations of either empirical features of multitasking behaviour or neurophysiological signals, and from there have deduced what kinds of algorithms could give rise to such multitasking phenomena. The last approach we shall discuss in our taxonomy uses specific knowledge about how neurons function, or are assumed to function, and generates networks that give rise to multitasking costs, typically as a consequence of some other computational feature of the system. For example, by leveraging what we know about synaptic activity, and more specifically the contribution of N-methyl-D-aspartate (NMDA) and a-Amino-3-hydroxy-5-methyl-4-isoxazolepropionic acid (AMPA) receptors to whether or not a given neuron fires, Zylberberg et al (2010) built a spiking neuron network model to perform sensorimotor tasks. Their network comprised local pools of sensory and motor neurons that converged on a common set of integrator neurons. They showed that sensory modules (e.g. the neurons that comprise the processors of sensory information input from different modalities) could maintain and amplify incoming information when receiving non-specific feedback from integrator neurons; i.e. excitatory inputs from integrator neurons act to boost and extend the duration of local, recurrent activity of whichever sensory pool it is feeding back to, thus maintaining a representation of the stimulus in the system, beyond the duration for which the stimulus is actually present. This ongoing activity has been proposed to form the basis of conscious access, or phenomenological experience (Dehaene et al., 1998; Mashour et al., 2020). Subsets of these integrator neurons link sensory and motor pools, and receive inputs from task-set neurons (see Figure 4A). Therefore the same integrator neurons also serve as detectors of the conjunction of a specific task-relevant stimulus-response pairing. For this model to perform sensorimotor functions successfully, response-execution neurons must inhibit the integrator neurons immediately after the response, in order to 
maintain a single response mode state and to avoid perseverative errors. The network performed multitasking with the aid of task-setting neurons that, just prior to the presentation of the first task, act to excite integrator neurons that connect the task 1 relevant sensory and motor neurons, and to inhibit briefly, those integrator neurons that connect the peripheral pools for task 2 . The construction of this network was an impressive feat, utilising 20,000 neurons and 46,000,000 synaptic connections.

During simulations of task performance, the network showed several features that were characteristic of findings from the empirical literature; first, multiple sensory and motor pools could be active at any given time, which is in accordance with the observation that sensory and motor information can be decoded from associated brain regions in parallel (Marti et al., 2015), and that sensory responses to task 2 inputs occur within a few hundred milliseconds, even when task 1 is underway (Marti et al., 2012; Sigman \& Dehaene, 2008). Moreover, the model showed that integrator neurons begin sharing information only after $300 \mathrm{~ms}$, and that their onset is delayed for task 2. Analogously, the P300, a component that is assumed to originate from frontal and parietal cortices and is often observed in neural signals recorded with high temporal resolution (e.g. MEG or electroencephalography, EEG) during sensorimotor processing, shows a typical onset of around $300 \mathrm{~ms}$, and a delayed onset for task 2 (Brisson \& Bourassa, 2014; Hesselmann et al., 2011; Marti et al., 2012; Sergent et al., 2005; Sigman \& Dehaene, 2008). Therefore, not only is this model grounded in known principles of neural function, it also generates many of the observed neural correlates of multitasking.

Interestingly, this model and the convergent empirical observations map neatly onto the originally proposed bottleneck model discussed earlier in this chapter, in that sensory and motor processes can proceed in parallel, and that delays occur at the information processing stage that maps a stimulus input to the task-relevant output. It also sheds some unique answers into the why's of multitasking limitations. For example, this system is solving the problem of how to maintain internal representations of external sensory inputs, something that is critical in a dynamic environment, and at a cost, engages an all-purpose recurrent system for maintaining internal activity that acts serially to preserve internal states. Moreover, this model solves the problem of how to integrate information between disparate sources, such as sensory inputs and motoric outputs, while maintaining stable internal states. The expense of which is a delayed, serial procedure of routing information between stimulus and response. Note that these explanations for why we see multitasking costs are compatible with the concepts of problem solving, representation and control that we discussed in the previous section. 

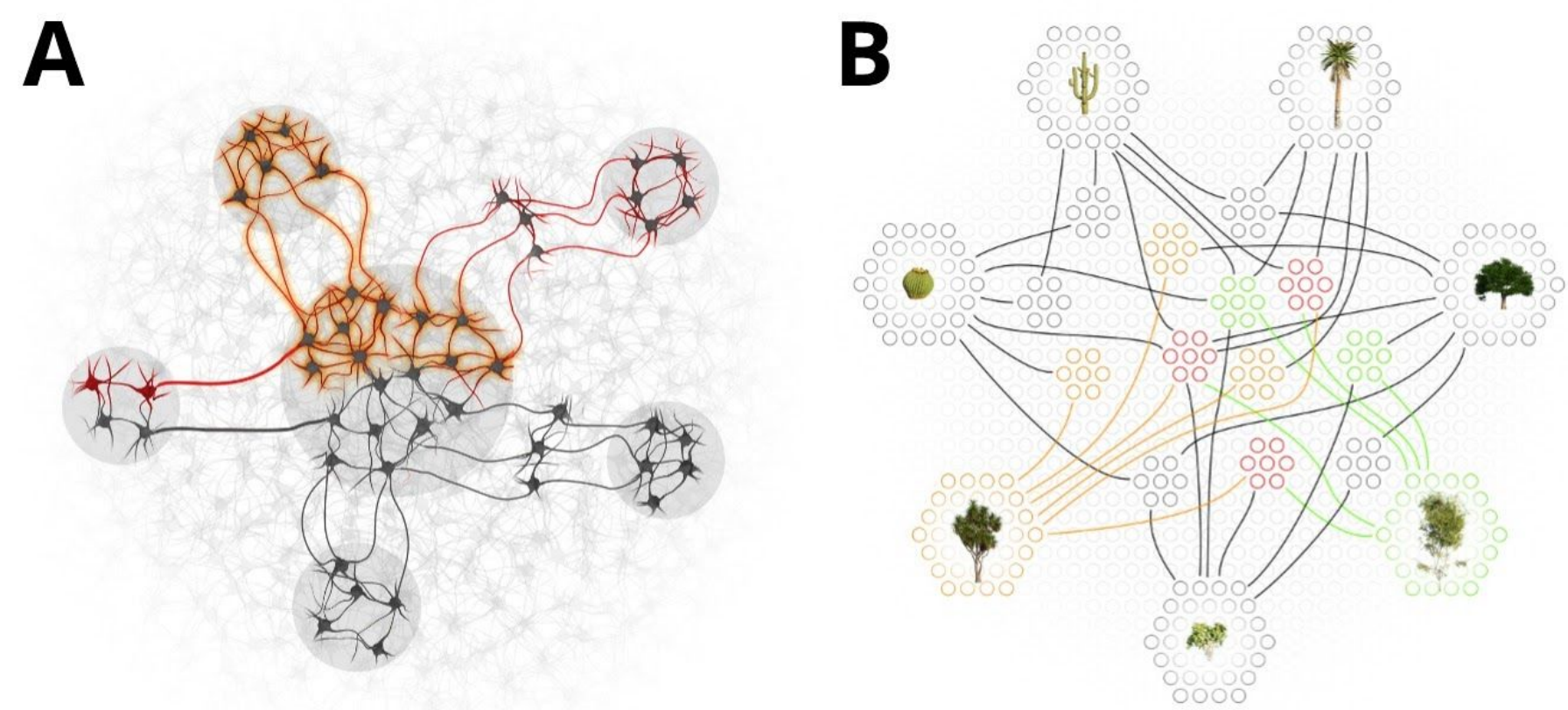

Figure 4: How modelling the activity of neural networks has leveraged insights into the causes of multitasking limitations. A) According to global workspace models, general purpose integrator neurons (central pool) send excitatory signals to maintain recurrent activity in local pools of sensory and motor neurons (peripheral pools). Stability of response-states requires temporary inhibition of integrator neurons, thereby causing delays to the completion of the second sensorimotor task. B) Overlapping representations in neural network models. Stimulus inputs overlap with hidden processing units to the extent that the stimuli share statistical regularities with one another. The consequent sharing in underlying stimulus-representations results can motivate more rapid learning of new tasks, but requires inhibitory mechanisms to control cross-talk in the network. The result is a limit on the number of operations that can be performed at any given time, independent of the size of the network.

\section{Neural Networks}

Recently, insights into the putative neural basis of multitasking have been made via the application of neural-network models to performance of the kinds of tasks that typically give rise to multitasking costs. The emerging picture from this work is that multitasking limits may not be due to some capacity limitation of an underlying neural resource, but rather may emerge as a consequence of a network that shares relevant information across task-contexts. Neural-network models are biologically inspired learning algorithms whose design is driven by the goal of mapping stimulus inputs to appropriate response outputs. The kind of tasks to which neural networks are typically applied include classification of natural images, natural language parsing, and time-series forecasting (Carpenter, 2001; Paliwal \& Kumar, 2009). Typically, a neural network model consists of an input vector, at least one hidden layer and an output vector. The input layer reflects 'neural activity' invoked by a stimulus input, the output layer reflects the response produced by the network given that stimulus, and the hidden layers reflect the system's internal representation of the stimulus. Each element of these vectors reflects a neural unit, whose strength of activation is reflected by the value of that element. Each vector connects to another 
via a weights matrix, which transforms its input into the activity of the next layer, typically via a nonlinear function. These weight matrices can be thought of as reflecting how the network has learned to transform the relevant inputs for representation at the next level in such a manner that the network can successfully represent the input and perform the task correctly. Over multiple iterations, the network is taught (for example via back propagation) to adjust these weights to achieve accuracy of performance. Although neural-networks do not necessarily reflect the exact computations performed by the brain's neurons to the same extent as the detailed spiking neuron network we discussed above, they do hold the potential for unravelling insightful and unexpected computational consequences given a system that seeks to transform inputs into correct outputs. For example, as we discuss below, the training of neural networks to perform the simple task of replicating recurring input values, which is assumed to be a proxy for a simple sensorimotor task, has demonstrated how a system may prefer to share representations between stimuli and tasks, insofar as they share featural or statistical overlap, and that this holds consequences for the extent to which that system can multitask without performance costs.

If we consider the structure of the world around us, we can immediately see both great variety and regularity. For example, your walk through a forest may be bordered by multiple tree species, each of which bears its own leaf type and fruit, but all consist of trunks, branches and leaves. It is not too great a stretch of the imagination to assume that it is wasteful for each tree species to be represented by sets of neurons that share no overlap, not to mention computationally impossible given the variety of stimuli in the world. Rather, subsets of neurons could contribute to the representations of multiple trees, according to the degree of featural overlap between exemplars. Such shared representations may potentially garner multiple benefits for any system that has to learn to perform multiple tasks in an information-rich world. It has been shown that the efficacy of machine learning algorithms is improved when a model is trained to simultaneously solve multiple problems from a single input, such as detecting both the gender and the emotional expression from the image of a face, relative to when the algorithm is trained on each task in isolation (e.g. Caruana, 1997). Moreover, novel tasks may be learned more rapidly when a new network is initialised with features derived from a network previously trained on multiple tasks (Yosinski et al., 2014). Shared representations motivate such learning by sharing informative features across overlapping tasks. This is protective, in that it prevents a system learning about the idiosyncrasies of incoming data, and it enables the system to develop informed priors over which elements of the new input are likely to contain useful data. Shared representations also support generalisation of learned information to subsequent tasks, resulting in a system that can acquire new tasks with greater ease than a system that does not share representations. Given that a remarkable feature of the human brain is the capacity to perform and learn a great range of tasks, any theory of the neural basis of multitasking that can provide answers as to why cognitive flexibility and limited multitasking capacity coexist as features of the human brain is indeed a compelling one.

It is hopefully clear why concurrently performing multiple tasks may suffer when there is competition for access to shared, underlying representations. Such a trade off between information sharing and multitasking ability has been demonstrated in neural-network models that have investigated how multitasking limitations may arise, not due to structural limitations, but rather as a consequence of the control mechanisms required to 
maintain task accuracy in a network that shares information across stimulus-response mappings (Feng et al., 2014) or as a consequence of exploiting statistical regularities between tasks to motivate rapid learning (Musslick et al., 2017). In the first, Feng et al (2014) sought to understand how the extent of information sharing in a neural-network may induce a control mechanism to titrate the activation strengths of given task inputs and outputs in order to maintain accuracy of stimulus-response mappings. The resulting activation strengths were taken as a proxy of how well the network could support multiple tasks concurrently. Specifically, the authors constructed a neural network model where pairs of input units were connected to pairs of output units to form a single 'task pathway'. Pairs of input-output units were selected to reflect neural representations of the two-alternative choice tasks commonly used in multitasking paradigms. To simulate the notion of shared representations, input units could not only connect to the output units of their own pathway, but also to the output units of other task pathways. These connections instantiate shared representations in the network. The total number of cross-talk connections was varied over simulations, as was the proportion that projected congruent weights to the task pathway with which they were connected. Congruent cross-talk acted to exaggerate the existing activity in the original task pathway. You could imagine this being analogous to seeing a red 'don't walk' light at a pedestrian crossing and a red light for your direction of road traffic. Both stimuli map to the same response of stopping. In contrast, incongruent cross-talk would antagonistically modulate activity in the task pathway. You could imagine a pedestrian starting to set foot onto the road as the traffic light turns green, even though the red 'don't walk' sign is still displayed. As with the incongruent cross-talk, the two signals compete to activate both responses of 'walk' and 'don't walk'.

The overarching goal of this neural-network was for each single task pathway to match the activity values of its output unit pair to the values of it's input unit pair, given the provided weights matrix of connectivity and shared representations. This is analogous to forming a correct stimulus-response mapping. To achieve this goal, a control mechanism was able to titrate input and output unit values. This mechanism constituted a vector, each element of which was a constant that scaled the input and output values of the corresponding pathway. The optimal control values were sought that resulted in the minimal mismatch between the activation values from the input and output vectors. Thus, this control mechanism serves to preserve the accuracy of stimulus-response mappings, in the presence of conflicting shared representations. The authors interpreted any resulting control values over a given value as reflecting tasks that had received sufficient activation for the task to be performed; i.e. these reflect active task pathways.

Of import, along with simulating the influence of the extent and congruence of cross-talk within the network, the authors also tested the effect of scaling up the network size (the tested range was 10-1000 pathways). This latter point is pertinent given the size of the human brain, and the large number of task pathways presumably required to support the goals of an average human. Interestingly, the simulation results revealed the following key features; as the proportion of incongruent irrelevant connections increased, the number of simultaneously activated task-pathways reduced. Indeed, as the network size increased, so did the rate of dropoff for simultaneous activations. In fact, regardless of the network size, the number of simultaneous active pathways 
converged to be approximately the same, when incongruency conditions were high. This result is interesting, because it suggests that even with a very large network, such as the human brain with its $\sim 85$ billion neurons (Herculano-Houzel, 2009), there should still be a striking and rapidly reached limit in the number of tasks that can be performed simultaneously when cross-talk is incongruent.

The above example shows that shared representations, insofar as they are reflected in cross connections between input and output nodes of a neural-network, can be regulated by a mechanism that preserves the accuracy of stimulus-response mappings by adjusting task activation strength. Although it is relatively easy to mentally deduce that sharing neural representations between common task elements may be a more efficient way for a system to operate, it is far more satisfying when empirical demonstrations uncover exactly what kind of gains may be accrued by a proposed principle of systemic organisation. In a subsequent conference paper, Musslick et al (2017) make in-roads to directly linking the observation that neural-networks learn new tasks more efficiently when sharing representations between tasks (Caruana, 1997; Yosinski et al., 2014) to the notion that this comes at the expense of multitasking ability. Again, the authors trained a neural-network to map stimulus inputs to given response outputs, although this time the exact mappings were determined by a randomly generated nonlinear function. Importantly, the network was trained to perform 9 tasks that mapped to shared output units. Specifically, 3 of 9 tasks would map to a common 5 of the 15 output units. Each task was required to activate only 1 of its possible 5 output units, given the stimulus input and the randomly generated function. Thus, this network contained two sources of input; a vector of stimulus input units, and a task vector of binary values reflecting which one of the 9 tasks was active on any given trial. Each input source was connected to a hidden associative layer by its own weights matrix. Both the associative and the task vectors connected to the output vector via separate weights matrices.

The authors trained the network under conditions that systematically varied overlap between task-relevant inputs and then tested multitasking accuracy as a function of that overlap. At one extreme, tasks relied on entirely separate stimulus inputs, and at the other, tasks that mapped onto different groups of response units were dependent on entirely the same input units. This is analogous to the same stimulus motivating different responses across contexts; for example the same ball could be kicked or thrown, depending on the requirements game being played at the time. By comparing the similarity of vectors from the weights matrix that reflects the learned connectivity between each task context to the associative layer, the authors were able to show that weights for tasks that shared stimulus inputs were more highly correlated than those that did not, even though they mapped to different response outputs. Correlation strength between these weight vectors served as a proxy for the extent of shared representations between tasks within the network.

When the network was required to perform two tasks in a multitask condition, there was a strong negative relationship between strength of shared representations and response accuracy, even though the two tasks to be performed were always mapped onto separate groups of response output units. Therefore, having tasks that share stimulus inputs motivates greater shared representation within the network, which results in a concomitant decrease in the ability to simultaneously perform two tasks drawing from that representation. At first the notion of 
shared inputs may appear to contradict observations that multitasking costs occur when stimulus inputs and response outputs do not overlap in modality. However, when considering more abstracted levels of the information processing hierarchy (Schmitz \& Duncan, 2018), it is fairly easy to see how inputs from different modalities would share some statistical overlap, and therefore at some point, can share an input into the circuit that gives rise to multitasking costs. For example, how often does a loud rumbling noise occur in conjunction with the sight of a large motorcycle or motor vehicle?

Musslick et al (2017) also tested how initialising the network with biases towards shared representations influenced how many iterations it took the network to learn the tasks. Less iterations were required when the task-to-associative layer connections were initially biased towards shared representations (i.e. starting weights were more highly correlated between tasks that shared stimulus inputs), relative to those that were biased against, replicating the finding that covarying internal representations facilitates learning across tasks that can use that representation. Interestingly, the number of iterations required to train the network was not only higher when the network was biased against representational similarity, but this also predicted multitasking accuracy; i.e. networks with lower covarying internal representations learn more slowly and multitask more accurately. Thus this neural-network model implies that there is a direct trade-off between learning and multitasking. Sharing representations fosters the acquisition of new tasks, at the decrement of multitasking ability. Interestingly, the same research group has also shown that an ideal Bayesian agent will sacrifice multitasking ability that could be achieved over the longer term, by selecting a shared representation scheme to learn tasks more rapidly in order to maximise reward gains in the shorter term (Sagiv et al., 2018), thereby corroborating the notion that more can be gained from organising a system to learn fast, and multitask less.

Overall, neural-network models teach us that the neural basis of multitasking may not be due to a structural limitation in long-range information sharing or limitations in the capacity of a central control mechanism (although, given the brain is finite in size, that there is some structural limit to its processing power is a fairly obvious implication), but rather may arise as a consequence of computational features of the network. Namely; a network that seeks to maximise learning can, as one solution, share stimulus-representations between tasks. This can invoke a requirement to exercise control over the excitation of concurrent stimulus-response mappings. This ensures that particular task pathways cannot dominate network activity due to their co-connectivity profile, and consequently reduce stimulus-response accuracy. Therefore, neural network models suggest that attenuated multitasking ability may actually be a consequence of the appropriate application of control. Moreover, where a system can leverage shared statistical structures to more rapidly acquire new abilities, a reduction in multitasking ability may be a necessary trade-off. Thus neurons that contribute codes to multiple task representations cannot contribute to all task representations at once, without activating inappropriate responses.

This avenue of investigating the neural basis of multitasking is exciting because it leverages focus away from how a capacity-limited processor could exist to give rise to capacity limits in multitasking; a line of thought that runs the risk of restating the problem in more technical terms. Instead this research demonstrates that multitasking limits could instead be a consequence of response regulation and effective learning. We also believe 
that the shared representations account may shed insights into why multitasking improves with practice, which we turn to below.

\section{Shared representations and the modification of the limits of multitasking: a novel perspective}

The neural network models above demonstrate how sharing representations across tasks may enable a system to acquire new tasks more rapidly. In our previous work (Garner \& Dux, 2015), we found that practice (extended learning) may lead to reduced overlap in the neurons recruited to perform each task, thereby increasing availability of computational power, or reducing potential crosstalk, to enable concurrent performance of the second task. The factors that drive this reduction in overlap remain to be elucidated. In this section, we seek to build links between the insights offered by the neural architectures perspective and our observations, which we have accrued from the system architectures perspective. By motivating new hypotheses, we seek to pinpoint the next steps to further our understanding of the neural basis of multitasking. To achieve this, we conducted a formal investigation into how multiple exposures to stimuli over different contexts could drive down shared representations between tasks. We first describe our model conceptually, in the section 'Reducing shared representations with practice'. We then elucidate the formalisation of our assumptions in the subsequent sections. Readers who wish to skip the technical details can proceed straight from the conceptual overview to the 'Simulating practice-related reductions in multitasking costs: new insights' section.

Reducing shared representations with practice

We assume that shared representations can occur at least in part as a consequence of initial overrepresentation of the task context, and that repeated exposures to this context serves to differentiate tasks, thereby reducing shared representations and consequently multitasking costs. Indeed, there is a large literature showing how context/environment influences learning across lower levels of information processing (Bouton, 2004; Gershman et al., 2010; Redish et al., 2007). By the task context, we mean everything that co-occurs with the task relevant stimulus at a given point in space and time. If we once again take a walk through the forest as an example, and we imagine that it is a new forest, full of entirely novel species, and that we seek to learn about one small tree that we know should bear delicious fruit (Figure 5A). Initially some of the trees will look very similar, for example, two types of trees may share so many common properties that we cannot tell whether they are two separate species, or a smaller and larger version of the same thing. Initially, the representation we have of the smaller tree serves as a good enough model for the larger tree (Figure 5C, first panel). However, as we walk through the forest, we get to see examples of the small tree in a multitude of contexts (Figure 5B). We learn which other trees are more or less likely to occur with our tree of interest, and which of the features are most probable, such as exact leaf shape or colour. After multiple exposures, extraneous contextual features get washed out so that we have a distinct representation of the small tree. At this point, our representation of the small tree is quite 
precise, and no longer serves as a reasonable model for the large tree. In fact at this point, it appears quite unlikely that they could be examples of the same species (Figure $5 \mathrm{C}$, last panel). This is the point at which we expect multitasking costs to be most largely attenuated. This is the basic idea behind our model that we specify below.
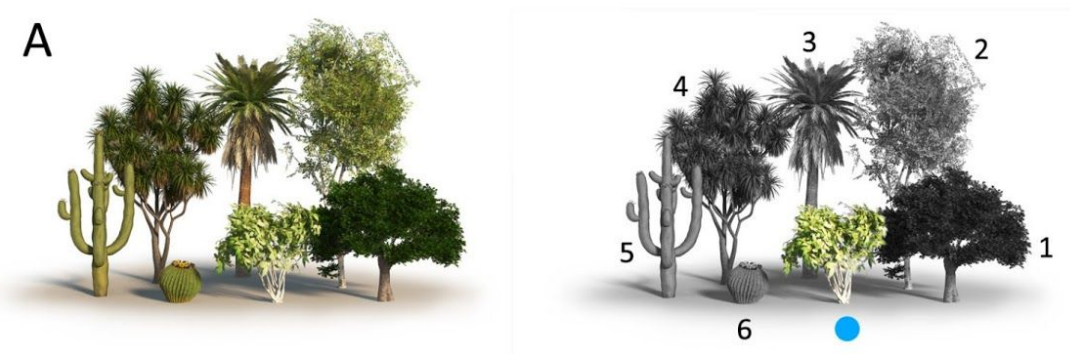

B

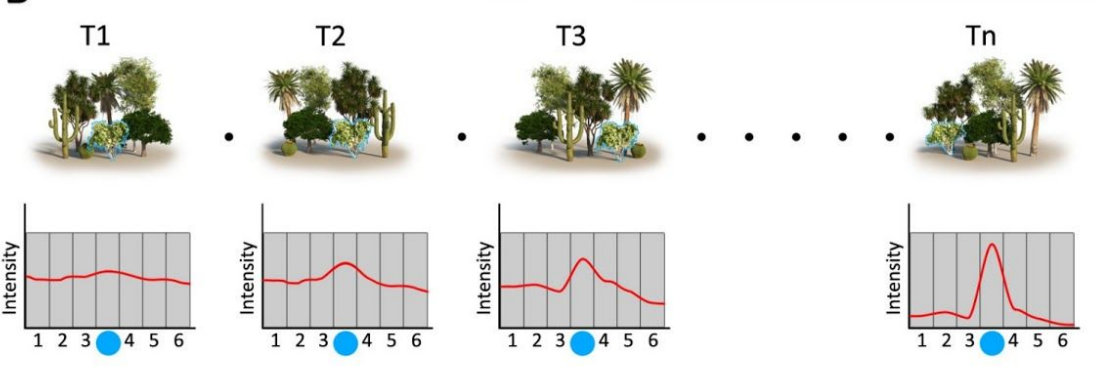

C
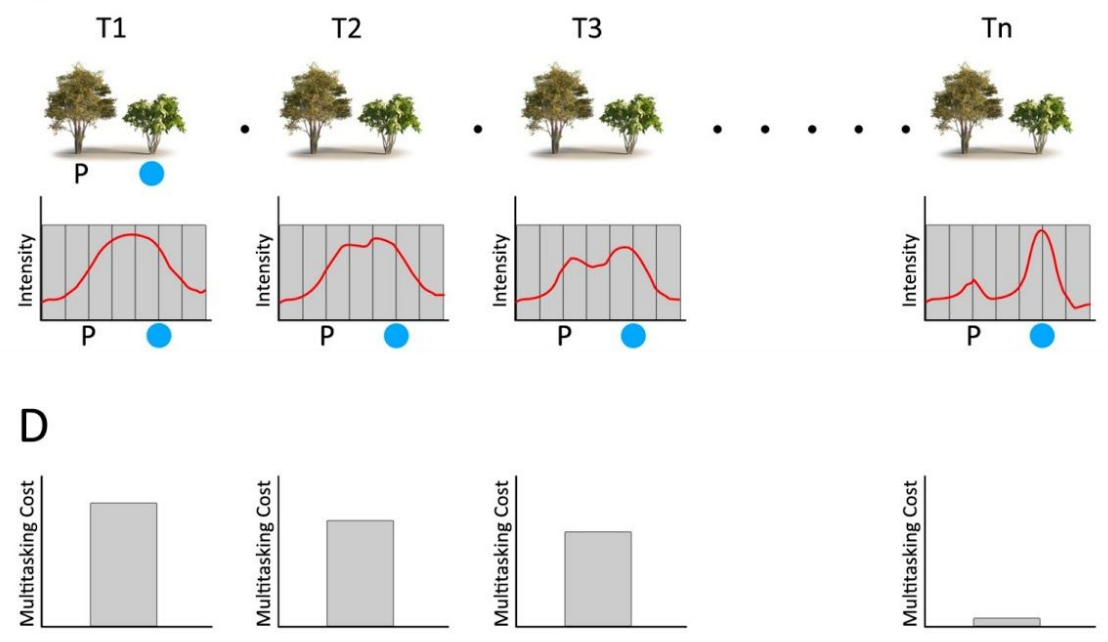
Figure 5: How practice reduces shared representations and consequently, multitasking costs. Panel A) A new forest, where the observer must learn about the tree marked with the blue dot (referred to here now as Tree X). B) At first, all trees are relatively new, thus the observer's model of Tree $X$ is quite undifferentiated from the other species present in the forest. However, multiple exposures to Tree $X$ across a variety of contexts serves to wash out extraneous, non-reliable information, resulting in a representation of the features that are highly specific to Tree X. C) As Tree $X$ is initially undifferentiated from Tree $P$, the representation of $X$ also serves as a reasonable representation of $P$. Thus the representations are highly shared, and any cognitive operations applied to both will result in multitasking costs (D). However, once the representation of Tree $X$ no longer serves as a good model for $P$, the representations are distinct and multitasking costs are reduced. $(T=$ time).

Internal representations of potential stimulus inputs

As we set up our investigation, we'll consider a simpler example than the one outlined above and one that is much closer to a set up that we would have in the lab. Lets consider a dual-task paradigm consisting of two sensory motor tasks (task $A$ and task $B$ ), where each task consists of 10 possible stimulus-response mappings. Therefore across the tasks there are 20 stimuli in total. We also assume that the agent knows that on some trials, one of 10 possible coincidental stimuli may occur. In real life this could be something like the experimenter coughing, or the light flickering in the lab. As we can instruct a participant as to the nature of the task, we assume that they have some internal model about what they expect to encounter during the task. We refer to this internal model as the internal representations of potential stimulus inputs. We represent this internal model as an $n \times p$ matrix where $n$ reflects the number of possible stimuli to be encountered across the two constituent tasks of the dual-task, including both the task-relevant and the 'coincidental' stimuli $(\mathrm{N}=30)$, and $p$ the number of featural elements that can be used to code each stimulus. Each dimension of $p$ can be thought of as a sensory quality, such as colour, shape, shadow, pitch etc. For our current purposes, we assume that each stimulus is encoded with 10 features. In accordance with previous observations that higher correlations between internal task representations motivates greater multitasking costs (Musslick et al., 2017; Sagiv et al., 2018), we also sought to investigate whether higher covariance between internal stimulus representations would influence the extent to which representations can be segregated with practice, and the consequences this has for reducing multitasking costs. To achieve this, we varied the covariance of the stimulus vectors that make up the internal template over two simulations (i.e. the vectors along $n$ ): in the first the stimulus vectors share low covariance with one another, and one where they share high covariance. We therefore construct the matrices so that stimulus vectors are randomly covarying with one another, within the range of $r=[-1,1]$ for low covariance, and $[9,11]$ for high covariance (these values were randomly selected).

Matching stimulus inputs to task templates

For each observation, or trial $t$, we use a vector $f=\left\langle f_{1}, f_{2}, \ldots, f_{n}\right\rangle$ to reflect the encoded stimulus input. Each dimension of the $f$ vector maps to the dimensions of $p$ in the agent's internal template. We assume that the 
encoding of each dimension of the stimulus vector $f$ is subject to additive noise $[N(0,0.1)]$ - i.e. to produce $f_{n}$, we take the vector that matches the agent's internal template of the randomly selected stimulus and add noise. This serves as the vector that represents the encoded stimulus. We then assume that on every trial, the agent determines which of its internal templates served as the best match for the stimulus input (i.e., response selection). To attain a measure of similarity $S$ between the sensory input $f$ and the internal representations of potential inputs (each $n$ ), we compute the reciprocal of the euclidean distance between the sensory input $f$ and the internal template for each stimulus $n$ :

$$
S=\frac{1}{\sqrt{\sum_{i=1}^{p}\left(f_{i}-n_{p i}\right)^{2}}}
$$

Updating the agents' internal model over trials

We assume that the agent expects that for each trial $t$, two tasks will be presented and that each task $T$ involves mapping 1 of 10 possible stimuli $s$ to the appropriate response. The agent should therefore construct a model over each task that verifies which stimuli map to that task space, i.e. which stimuli are most likely given the task context (e.g. the context of task B). Let's consider the model for task B; to determine which of the 30 possible outcomes (those for task A, B and the coincidental stimuli) are most likely given that the agent is in task B, we can use the Dirichlet distribution, which is a multivariate expansion of the binomial distribution, such that:

$$
p(s ; \alpha)=\operatorname{Dir}(\alpha)=\frac{\Gamma\left(\sum_{n=1}^{N} \alpha_{n}\right)}{\prod_{n=1}^{N} \Gamma\left(\alpha_{n}\right)} \prod_{n=1}^{N} s^{\alpha_{n}-1}
$$

Where $\alpha=\left[\alpha_{1}, \alpha_{2}, \ldots, \alpha_{n}\right]$, and $n$ is the number of total stimuli, both task-related and coincidental. Each $\alpha$ can be thought of as a proxy for the number of times that the stimulus is presented in the context of task $A$. We assume that the agent begins with no reason to believe that any stimulus will occur more frequently than any other, and assume initial uniform priors over all stimuli, i.e. $\alpha_{n}=1$. Each time a new stimulus is presented, the agent updates the probability for what stimulus was shown, given the task context. This is done by updating the alpha parameters of the Dirichlet distribution with the similarity value calculated for each stimulus input, such that:

$$
\alpha_{n_{t}}=\gamma\left(\alpha_{n_{t}-1}+S_{n}\right)
$$

where $\gamma$ is the learning rate, which for our purposes, we set $\gamma=.01$, and $S_{n}$ is the similarity metric between each internal representation and the stimulus input, as defined above.

We also assume that the agent seeks to exploit shared representations between tasks. Specifically, the agent will use the model for task $A$ as an approximation for task $B$, insofar as task $A$ provides information about $B$. This is analogous to the small tree serving as a reasonable model for the big tree, at the start of the walk through the forest. This can be quantified using the Kullback-Liebler $(\mathrm{KL})$ divergence measure, which is derived as follows 
for the Dirichlet distribution:

$K L(p \| q)=\log \Gamma\left(\alpha_{0}\right)-\sum_{k=1}^{K} \log \Gamma\left(\alpha_{k}\right)-\log \Gamma\left(\beta_{0}\right)+\sum_{k=1}^{K} \log \Gamma\left(\beta_{k}\right)+\sum_{k=1}^{K}\left(\alpha_{k}-\beta_{k}\right)\left(\psi\left(\alpha_{k}\right)-\psi\left(\alpha_{0}\right)\right)$

where $q$ is the model (or expectations of probable stimuli) over task $A, p$ the distribution over task $B$ (note that $\beta$ reflects the parameters for $q$, i.e. task $\mathrm{A}$, and $\alpha$ reflects the parameters for task $\mathrm{B}$ ).

We quantify the extent of dual task interference as proportional to the extent that $p$ (task B) can be encoded by $q$ (task A). Specifically, we reasoned that multitasking costs would be proportional to the extent that task $B$ can be explained by the model of task $A$, as this reflects the extent to which a representation of task $A$ can serve as a representation of task $B$. Therefore to approximate a duration of multitasking (MT) interference, we need a measure of the extent to which the model over task $A$ can serve to explain task $B$. To achieve that measure, we take the reciprocal of the $\mathrm{KL}$ divergence.

$$
M T=\frac{1}{K L(p \| q)}
$$

Simulating practice-related reductions in multitasking costs: new insights

We started by simulating what happens to predicted multitasking costs (MT) when the agent practices task $A$ and task $B$. To recap, task $A$ and task $B$ are each sensorimotor mapping tasks, where the agent must produce the correct response to 1 of 10 possible stimulus inputs. Practicing this task over time is our analogy for walking through the forest and being exposed to multiple instances of the same tree. For task $A$ and task $B$, we assume that the task relevant stimuli occur on every trial, and where 1 of 10 possible coincidental stimuli occur on every other trial. We reran each simulation 1000 times, to attain confidence estimates on the predicted multitasking costs. We repeated this process separately for internal representations that share high and low covariance; i.e. are more or less likely to be encodable with shared representations. The results are presented in Figure 6A. As can be seen, this model produces a learning curve that is similar as to what would be expected in a typical multitasking practice study (see Figure 6B). Interestingly, differences in the extent of covariance between stimulus representations in the internal task set affects the asymptote of the multitasking costs in a largely additive manner, and therefore ultimately places a limit on the extent to which multitasking costs can be attenuated. We have observed something similar in our own practice data (Garner, Tombu \& Dux, 2014). For example, when participants practice single sensorimotor tasks over thousands of trials, they are slower when selecting between coloured discs than they are when selecting between letters, and this slowing is additive (Figure 6C). Although no formal analysis has been applied, it is arguable that the coloured discs were more similar to one another (shared higher covariance) than the letters. However, it is important to note that we have only tested two arbitrarily chosen covariance ranges and further investigations are necessary to see if this observed relationship holds over multiple 
covariance contexts. Moreover, we have only considered a scenario where there is no stimulus overlap between tasks $A$ and $B$. Task sets and consequent stimulus displays are typically more complex, consisting of multiple stimuli and features, some of which are relevant across task contexts. For example, a target may be presented inside a circular placeholder in both task $A$ and task $B$, or the upcoming presentation of both tasks may be preceded by a common alerting cue. It would be interesting to determine the extent to which overlapping contexts influence the extent to which the experience of task $A$ can serve as a model for task $B$, and the consequent impact for multitasking costs.

Our model shows that multitasking costs between novel tasks may be due at least in part to an initial and extraneous over sharing between the multidimensional encoding of sensory inputs to response outputs. Specifically, initial uncertainty about the task space can result in the leveraging of alternate encoding routines, insofar as they provide a sufficiently useful model for current task performance. Interestingly, this investigation makes new predictions; multitasking performance improvements should occur as a function of the ongoing covariance between task spaces, and asymptotes reached in response to stable task states should be a linear function of the covariance between feature dimensions used to encode the tasks. Given the potential additive nature of the relationship between task covariances and performance asymptotes, we postulate that perhaps multitasking costs can be related in a novel way to the Hick-Hyman law of decision time (Hick, 1952; Hyman, 1953). This law states that the time taken to perform a sensorimotor task is a negative linear function of the information offered (in bits) by the stimulus. Here we suggest that multitasking costs may be a positive linear function of the information offered between task-spaces. It remains to be determined what constitutes a unit of information in the brain's representation of the task space, and we return to this point in the conclusions below.

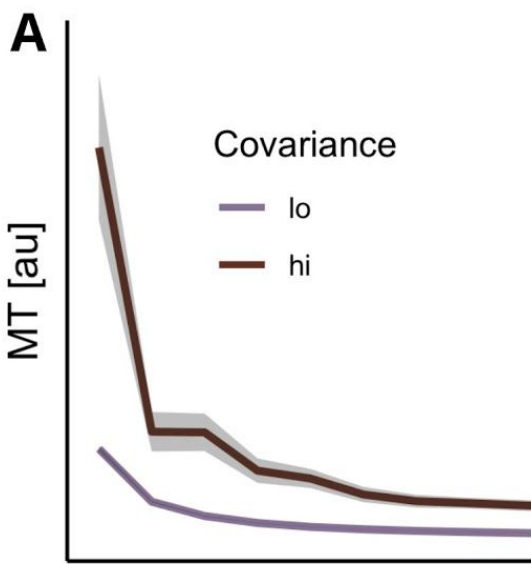

trials [au]
B

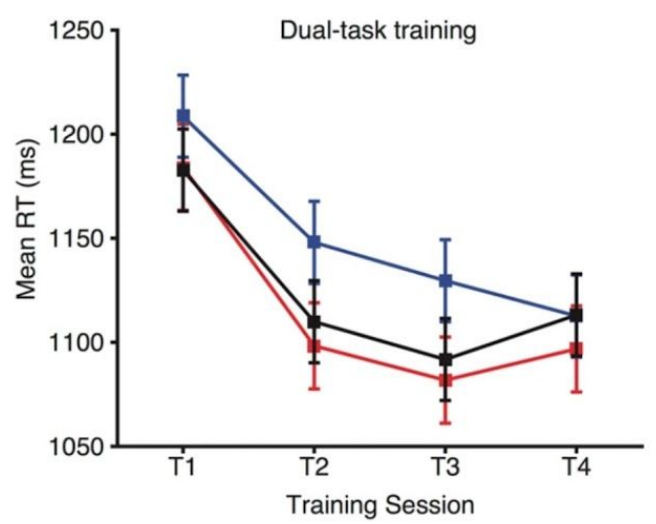

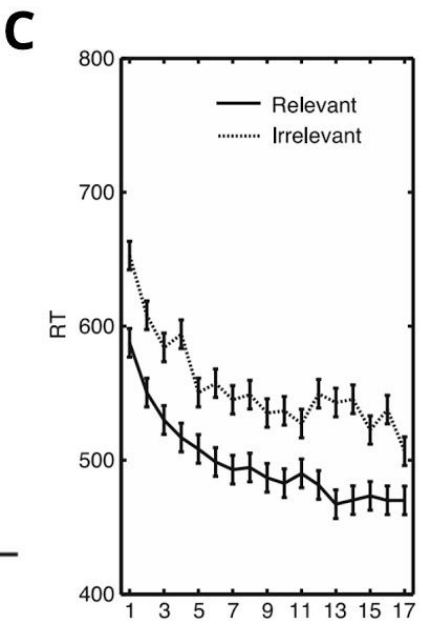


Figure 6. Model predictions and observed data. A) Multitasking costs (MT) diminish as the task contexts become more differentiated over multiple exposures (i.e. the probability of any given stimulus, given the context of task $A$ ceases to serve as a reasonable model for the probability of a given stimulus, given the context of task $B$. Multitasking costs remained higher when the covariance between stimulus representations was high (hi), relative to low (lo). The predicted reductions in MT costs look similar to previous observations, for example B) where participants completed a multitasking paradigm over multiple training sessions under differing brain stimulation conditions (Filmer, Lyons, et al., 2017). Additionally, the observed increase in costs for high relative to low covariances in stimulus representations looks analogous to previous observations that even over thousands of trials, participants take longer to respond to stimuli that potentially share higher covariances (panel C, Irrelevant stimuli) relative to those sharing lower covariances (Relevant stimuli). Panel C is taken from (Garner et al., 2014).

\section{Conclusions and future directions}

In our taxonomy of the investigations into the neural basis of multitasking, we have found that there are three broad approaches to tackling this question, pertaining to whether theorising occurs at the level of cognitive, system, or neural architectures. The first involves taking proposed cognitive architectures and seeking to map them to observable neural correlates. The second uses observations of systems-level neural activity to ask how a system that shows those features could perform tasks that incur multitasking costs. The third involves building networks of neural-like or neural-inspired units and seeking how multitasking costs arise as a consequence of the computational features of the system. These approaches have yielded multiple insights into the hows and whys of the neural basis of multitasking. Here we now seek to highlight points of convergence and disparity between the three approaches and identify the next steps required to further our understanding, using Marr's (1982) levels of understanding as our framework for discussion.

\section{Understanding why: what problem is the brain trying to solve?}

Interestingly, understanding the why of multitasking limitations and their neural underpinnings is difficult to pin down definitively. Just as the interrogation of the neural basis of multitasking can occur at multiple levels of analysis, from verbal descriptions of cognitive stages down to the formalised actions of synapses, so too can the answer as to why the brain so often shows multitasking limitations. For example, the implications of the central bottleneck model are that the brain is limited at certain stages of information processing, which while evidently true, does not shed insights into why the brain shows such limitations. Indeed, sometimes this literature is interpreted as suggesting that this limitation may be undesirable, and should be viewed as something to be overcome (Maquestiaux et al., 2008; Maquestiaux et al., 2010). Multitasking costs increase with aging (Maquestiaux et al., 2004) and multiple efforts have been leveraged at attenuating them in older adults (Anguera et al., 2013; Maquestiaux et al., 2004; Strobach et al., 2012b, 2012a). Meanwhile, proposed 'executive' algorithms such as task scheduling or resource management imply that multitasking costs are evident in human behaviour because this is the optimal way to ensure successful task completion in complex environments. The notion that multitasking costs may result because the brain has opted to optimise for something other than multitasking 
performance is echoed in models of complex problem solving, and network models that seek to mimic multitasking costs. Namely, according to the former, complex problems are best solved by being broken down into subroutines that are executed sequentially, however, why exactly some subroutines cannot be executed concurrently remains to be elucidated. Note: these models were never developed to explain why multitasking limits exist, but why they may be applicable remains evident. What network models that seek to mimic multitasking costs tell us is that a neural network that shares information across task representations will learn new tasks more quickly and may even accrue greater reward over the long term, relative to a system that has engaged in parallelism. Moreover, the notion of multitasking costs stemming from shared underlying representations suggests that multitasking costs may be a necessary function of a system that builds internal models of a world that contains statistical dependencies. Thus the expense of our remarkably flexible, accurate and adaptable behavioural repertoires may be the capacity to multitask without cost.

\section{What are the computational algorithms underlying multitasking performance?}

Of the proposed and implicated computational algorithms discussed in this chapter, we see two key emergent themes in relation to multitasking; those of organisation and representation. Central executive models stemming from the cognitive architectures approach imply that multitasking costs emerge when a system is required to schedule access to functional modules that perform elements of constituent tasks. Thus multitasking occurs as a function of scheduling. The notion of subroutines is indeed a pervasive one across investigations into aspects of cognition that require some element of control (e.g. Duncan, 2010; Meyer \& Kieras, 1997; Roelfsema et al., 2000). The study of system architectures has suggested that a common frontal-parietal system may encode the contents of the current subgoal, and that a serial processing strategy may act to preserve the appropriate response required to attain that subgoal. Thus multitasking costs stem from the sequential suppression of competing responses. Moreover, given that both the study of system and neural architectures implies that a common pool of neurons are recruited to encode the current subgoal, a serial representation strategy may arise in order to preserve the integrity of representations. Any flexible and adaptive system requires a method to ensure that subgoal representations remain distinct, which would no doubt be challenging when units capable of representing any subgoal are concurrently recruited to represent multiple subgoals. Taken together, these findings imply that multitasking costs may play a role in the preservation of causality judgements. For example, if two actions are selected in parallel, how would a system apply sensory feedback to credit which action caused the successful attainment of each subgoal? Rather, by ensuring a schedule of response-selection, sensory feedback can be credited to the action with a greater likelihood of accuracy. Whether this is indeed the case remains an open question.

Another point of convergence between the system and neural architecture approaches is the finding that systems are likely to share information between task representations. There is broad support that the brain gains representational efficiency by sharing representations between tasks, and that this occurs at the direct expense of multitasking capacity. In the case of the systems approach to the study of human cognition, the emerging picture 
is that the subcortical and associative cortices contain neurons that carry information regarding current task set rules, across multiple tasks - i.e. an internal model of the current statistical dependencies is required to perform a given subgoal. At first it may appear perplexing that given the exact number of neurons in this scenario, that we could reach a point of hardware overload that could induce multitasking costs. Interestingly, what we have found from the application of neural network models is that a system of any size that shares information between processing units (i.e. neurons) will converge on being able to perform only a few tasks simultaneously. Thus a system that shares information between tasks achieves flexibility and adaptability, but this occurs at the expense of parallel processing. These findings therefore imply that statistical dependencies between task contexts provide a hard limit on multitasking performance when a system engages in information sharing. What remains to be discovered relates to exactly how a system may represent a task space that enables information sharing. Specifically, what are the dimensions along which the brain encodes task states? Which dimensions impact most on information sharing, and hence, multitasking? Is information sharing between tasks determined by continuous or piecewise functions? The answers to such questions will not only provide further insights into the neural basis of multitasking, but also the representational units that make up the neural states that support adaptive cognition.

What are the physical implementations of these algorithms?

Investigations into the neural architectures that underpin multitasking performance have yielded promising advances for understanding potential hardware implementations of the observable cognitive phenomena. Models of neural architectures containing biologically plausible synaptic dynamics have shown how neurons may integrate information between sensory modalities, and how successful integration may require a serial processing strategy. The next exciting steps are to extend neural network architectures that support shared representations to implement neurophysiologically plausible rules for synaptic dynamics, and to determine whether multitasking capacity is similarly rapidly attenuated in such a system. This would link a physical implementation to a putative algorithm that drives multitasking limitations; namely the transformation of high dimensional data (i.e. all incoming sensory data from the environment) into latent factors that describe the external world, and thereby multiple task contexts, in a lower dimensional space. According to this view, units representing shared latent factors will cause greater multitasking costs than those that do not. Demonstrating a biologically plausible architecture that instantiates these principles would be an exciting and critical advance for the shared representations theory.

The effort of understanding the hardware implementation of multitasking operations can also be informed and constrained by the insights offered by the cognitive and system architecture approaches. For example, the LPFC has consistently been implicated as one site of potential importance. We may be able to next identify whether the LPFC neurons that appear to represent information pertaining to constituent tasks differ from their neighbours in terms of genetic expression, structure, connectivity and dynamics, which will motivate new inferences regarding the capacities of a system that incorporates these elements into its computational principles. Beyond the LPFC, investigations have largely shown that a set of distributed frontal-parietal brain regions and the striatum have been linked to multitasking costs. The striatum contains multiple cell types, containing differing dopamine, cholinergic and GABAergic receptors (Tepper \& Plenz, 2006) that have been linked to various modes 
of learning, such as prediction error updating and classical conditioning (Cox \& Witten, 2019). Future investigations can leverage these observations to make formal links between the logic underlying synaptic dynamics and representation sharing in the interface between the striatum and the cortex. Such efforts, while enormously challenging, will hopefully feedback to constrain putative mechanisms of neural computation, thereby making the next critical steps required for understanding the neural basis of multitasking.

\section{References}

Anguera, J. A., Boccanfuso, J., Rintoul, J. L., Al-Hashimi, O., Faraji, F., Janowich, J., Kong, E., Larraburo, Y., Rolle, C., Johnston, E., \& Gazzaley, A. (2013). Video game training enhances cognitive control in older adults. Nature, 501(7465), 97-101.

Ashby, F. G., Turner, B. O., \& Horvitz, J. C. (2010). Cortical and basal ganglia contributions to habit learning and automaticity. Trends in Cognitive Sciences, 14(5), 208-215.

Bornstein, A. M., \& Daw, N. D. (2011). Multiplicity of control in the basal ganglia: computational roles of striatal subregions. Current Opinion in Neurobiology, 21(3), 374-380.

Bouton, M. E. (2004). Context and behavioral processes in extinction. Learning \& Memory , 11(5), $485-494$.

Bratzke, D., \& Janczyk, M. (2020). Introspection about backward crosstalk in dual-task performance. Psychological Research. https://doi.org/10.1007/s00426-019-01282-3

Bratzke, D., Rolke, B., \& Ulrich, R. (2009). The source of execution-related dual-task interference: motor bottleneck or response monitoring? Journal of Experimental Psychology. Human Perception and Performance, 35(5), 1413-1426.

Brisson, B., \& Bourassa, M. Ė. (2014). Masking of a first target in the attentional blink attenuates the P 3 to the first target and delays the P 3 to the second target. Psychophysiology. https://onlinelibrary.wiley.com/doi/abs/10.1111/psyp.12204

Brown, S. D., \& Heathcote, A. (2008). The simplest complete model of choice response time: linear ballistic accumulation. Cognitive Psychology, 57(3), 153-178.

Caballero, J. A., Humphries, M. D., \& Gurney, K. N. (2018). A probabilistic, distributed, recursive mechanism for decision-making in the brain. PLoS Computational Biology, 14(4), e1006033.

Camilleri, J. A., Müller, V. I., Fox, P., Laird, A. R., Hoffstaedter, F., Kalenscher, T., \& Eickhoff, S. B. (2018). Definition and characterization of an extended multiple-demand network. Neurolmage, 165, 138-147.

Carpenter, G. A. (2001). Neural-network models of learning and memory: leading questions and an emerging framework. Trends in Cognitive Sciences, 5(3), 114-118.

Caruana, R. (1997). Multitask Learning. Machine Learning, 28(1), 41-75.

Cho, K. W., Altarriba, J., \& Popiel, M. (2015). Mental juggling: when does multitasking impair reading comprehension? The Journal of General Psychology, 142(2), 90-105.

Collette, F., Olivier, L., Van der Linden, M., Laureys, S., Delfiore, G., Luxen, A., \& Salmon, E. (2005). Involvement of both prefrontal and inferior parietal cortex in dual-task performance. Brain Research. Cognitive Brain Research, 24(2), 237-251.

Cox, J., \& Witten, I. B. (2019). Striatal circuits for reward learning and decision-making. Nature Reviews. Neuroscience. https://doi.org/10.1038/s41583-019-0189-2

Dayan, P., \& Abbott, L. F. (2001). Theoretical Neuroscience: Computational and Mathematical Modeling of Neural Systems. MIT Press.

Dehaene, S., Kerszberg, M., \& Changeux, J. P. (1998). A neuronal model of a global workspace in effortful cognitive tasks. Proceedings of the National Academy of Sciences of the United States of America, 95(24), 14529-14534.

Dreher, J.-C., \& Grafman, J. (2003). Dissociating the roles of the rostral anterior cingulate and the lateral prefrontal cortices in performing two tasks simultaneously or successively. Cerebral Cortex , 13(4), 329-339.

Duncan, J. (2010). The multiple-demand (MD) system of the primate brain: mental programs for intelligent behaviour. Trends in Cognitive Sciences, 14(4), 172-179. 
Duncan, J. (2013). The structure of cognition: attentional episodes in mind and brain. Neuron, 80(1), 35-50.

Dux, P. E., Ivanoff, J., Asplund, C. L., \& Marois, R. (2006). Isolation of a central bottleneck of information processing with time-resolved FMRI. Neuron, 52(6), 1109-1120.

Dux, P. E., Tombu, M. N., Harrison, S., Rogers, B. P., Tong, F., \& Marois, R. (2009). Training improves multitasking performance by increasing the speed of information processing in human prefrontal cortex. Neuron, 63(1), 127-138.

Erickson, K. I., Colcombe, S. J., Wadhwa, R., Bherer, L., Peterson, M. S., Scalf, P. E., \& Kramer, A. F. (2005). Neural correlates of dual-task performance after minimizing task-preparation. Neurolmage, 28(4), 967-979.

Feng, S. F., Schwemmer, M., Gershman, S. J., \& Cohen, J. D. (2014). Multitasking versus multiplexing: Toward a normative account of limitations in the simultaneous execution of control-demanding behaviors. Cognitive, Affective \& Behavioral Neuroscience, 14(1), 129-146.

Filmer, H. L., Lyons, M., Mattingley, J. B., \& Dux, P. E. (2017). Anodal tDCS applied during multitasking training leads to transferable performance gains. Scientific Reports, 7(1), 12988.

Filmer, H. L., Mattingley, J. B., Marois, R., \& Dux, P. E. (2013). Disrupting prefrontal cortex prevents performance gains from sensory-motor training. The Journal of Neuroscience: The Official Journal of the Society for Neuroscience, 33(47), 18654-18660.

Filmer, H. L., Varghese, E., Hawkins, G. E., Mattingley, J. B., \& Dux, P. E. (2017). Improvements in Attention and Decision-Making Following Combined Behavioral Training and Brain Stimulation. Cerebral Cortex , 27(7), 3675-3682.

Friston, K. J., Harrison, L., \& Penny, W. (2003). Dynamic causal modelling. Neurolmage, 19(4), $1273-1302$.

Garner, K., Garrido, M. I., \& Dux, P. E. (2020). Cognitive capacity limits are remediated by practice-induced plasticity between the Putamen and Pre-Supplementary Motor Area. In bioRxiv (p. 564450). https://doi.org/10.1101/564450

Garner, K. G., \& Dux, P. E. (2015). Training conquers multitasking costs by dividing task representations in the frontoparietal-subcortical system. Proceedings of the National Academy of Sciences of the United States of America, 112(46), 14372-14377.

Garner, K. G., Tombu, M. N., \& Dux, P. E. (2014). The influence of training on the attentional blink and psychological refractory period. Attention, Perception \& Psychophysics, 76(4), 979-999.

Gershman, S. J., Blei, D. M., \& Niv, Y. (2010). Context, learning, and extinction. Psychological Review, 117(1), 197-209.

Graybiel, A. M., \& Grafton, S. T. (2015). The striatum: where skills and habits meet. Cold Spring Harbor Perspectives in Biology, 7(8), a021691.

Gurney, K., Prescott, T. J., \& Redgrave, P. (2001). A computational model of action selection in the basal ganglia. I. A new functional anatomy. Biological Cybernetics, 84(6), 401-410.

Haxby, J. V. (2012). Multivariate pattern analysis of fMRI: the early beginnings. Neurolmage, 62(2), 852-855.

Hazeltine, E., \& Ruthruff, E. (2006). Modality pairing effects and the response selection bottleneck. Psychological Research, 70(6), 504-513.

Hazeltine, E., Ruthruff, E., \& Remington, R. W. (2006). The role of input and output modality pairings in dual-task performance: evidence for content-dependent central interference. Cognitive Psychology, 52(4), 291-345.

Hélie, S., Ell, S. W., \& Ashby, F. G. (2015). Learning robust cortico-cortical associations with the basal ganglia: an integrative review. Cortex; a Journal Devoted to the Study of the Nervous System and Behavior, 64, 123-135.

Herculano-Houzel, S. (2009). The human brain in numbers: a linearly scaled-up primate brain. Frontiers in Human Neuroscience, 3, 31.

Hesselmann, G., Flandin, G., \& Dehaene, S. (2011). Probing the cortical network underlying the psychological refractory period: a combined EEG-fMRI study. Neurolmage, 56(3), 1608-1621.

Hick, W. E. (1952). On the rate of gain of information. The Quarterly Journal of Experimental Psychology, 4(1), 11-26.

Huestegge, L., \& Koch, I. (2010). Crossmodal action selection: evidence from dual-task compatibility. Memory \& Cognition, 38(4), 493-501.

Hyman, R. (1953). Stimulus information as a determinant of reaction time. Journal of Experimental Psychology, 45(3), 188-196.

Jackson, J. B., \& Woolgar, A. (2018). Adaptive coding in the human brain: Distinct object features are encoded by 
overlapping voxels in frontoparietal cortex. Cortex; a Journal Devoted to the Study of the Nervous System and Behavior, 108, 25-34.

Jackson, J., Rich, A. N., Williams, M. A., \& Woolgar, A. (2017). Feature-selective Attention in Frontoparietal Cortex: Multivoxel Codes Adjust to Prioritize Task-relevant Information. Journal of Cognitive Neuroscience, 29(2), 310-321.

Jahanshahi, M., Obeso, I., Rothwell, J. C., \& Obeso, J. A. (2015). A fronto-striato-subthalamic-pallidal network for goal-directed and habitual inhibition. Nature Reviews. Neuroscience, 16(12), 719-732.

Janczyk, M., Renas, S., \& Durst, M. (2018). Identifying the locus of compatibility-based backward crosstalk: Evidence from an extended PRP paradigm. Journal of Experimental Psychology. Human Perception and Performance, 44(2), 261-276.

Jiang, Y., Saxe, R., \& Kanwisher, N. (2004). Functional magnetic resonance imaging provides new constraints on theories of the psychological refractory period. Psychological Science, 15(6), 390-396.

Joel, D., Niv, Y., \& Ruppin, E. (2002). Actor-critic models of the basal ganglia: new anatomical and computational perspectives. Neural Networks: The Official Journal of the International Neural Network Society, 15(4-6), 535-547.

Johnston, J. C., \& McCann, R. S. (2006). On the locus of dual-task interference: Is there a bottleneck at the stimulus classification stage? Quarterly Journal of Experimental Psychology , 59(4), 694-719.

Klapp, S. T., Maslovat, D., \& Jagacinski, R. J. (2019). The bottleneck of the psychological refractory period effect involves timing of response initiation rather than response selection. Psychonomic Bulletin \& Review, 26(1), $29-47$.

Ko, Y.-T., \& Miller, J. (2014). Locus of backward crosstalk effects on task 1 in a psychological refractory period task. Experimental Psychology, 61(1), 30-37.

Lebedev, M. A., Messinger, A., Kralik, J. D., \& Wise, S. P. (2004). Representation of attended versus remembered locations in prefrontal cortex. PLoS Biology, 2(11), e365.

Levy, J., \& Pashler, H. (2008). Task prioritisation in multitasking during driving: opportunity to abort a concurrent task does not insulate braking responses from dual-task slowing. Applied Cognitive Psychology, 22(4), 507-525.

Logan, G. D., \& Gordon, R. D. (2001). Executive control of visual attention in dual-task situations. Psychological Review, 108(2), 393-434.

Logothetis, N. K. (2008). What we can do and what we cannot do with fMRI. Nature, 453(7197), 869-878.

Maquestiaux, F., Hartley, A. A., \& Bertsch, J. (2004). Can practice overcome age-related differences in the psychological refractory period effect? Psychology and Aging, 19(4), 649-667.

Maquestiaux, F., Laguë-Beauvais, M., \& Bherer, L. (2008). Bypassing the central bottleneck after single-task practice in the psychological refractory period paradigm: Evidence for task automatization and greedy resource .... Memory . https://link.springer.com/article/10.3758/MC.36.7.1262

Maquestiaux, F., Laguë-Beauvais, M., Ruthruff, E., Hartley, A., \& Bherer, L. (2010). Learning to bypass the central bottleneck: declining automaticity with advancing age. Psychology and Aging, 25(1), 177-192.

Marois, R., \& Ivanoff, J. (2005). Capacity limits of information processing in the brain. Trends in Cognitive Sciences, 9(6), 296-305.

Marois, R., Larson, J. M., Chun, M. M., \& Shima, D. (2006). Response-specific sources of dual-task interference in human pre-motor cortex. Psychological Research, 70(6), 436-447.

Marr, D. (1982). Vision: A Computational Investigation into the Human Representation and Processing of Visual Information. http://papers.cumincad.org/cgi-bin/works/_id=ecaade2013/Show?fafa

Marti, S., King, J.-R., \& Dehaene, S. (2015). Time-Resolved Decoding of Two Processing Chains during Dual-Task Interference. Neuron, 88(6), 1297-1307.

Marti, S., Sigman, M., \& Dehaene, S. (2012). A shared cortical bottleneck underlying Attentional Blink and Psychological Refractory Period. Neurolmage, 59(3), 2883-2898.

Mashour, G. A., Roelfsema, P., Changeux, J.-P., \& Dehaene, S. (2020). Conscious Processing and the Global Neuronal Workspace Hypothesis. Neuron, 105(5), 776-798.

Messinger, A., Lebedev, M. A., Kralik, J. D., \& Wise, S. P. (2009). Multitasking of attention and memory functions in the primate prefrontal cortex. The Journal of Neuroscience: The Official Journal of the Society for Neuroscience, 29(17), 5640-5653. 
Meyer, D. E., \& Kieras, D. E. (1997). A computational theory of executive cognitive processes and multiple-task performance: Part 1. Basic mechanisms. Psychological Review, 104(1), 3-65.

Meyer, D. E., \& Kieras, D. E. (1997). A computational theory of executive cognitive processes and multiple-task performance: Part 2. Accounts of psychological refractory-period phenomena. Psychological Review, 104(4), 749-791.

Meyers, E. M., Freedman, D. J., Kreiman, G., Miller, E. K., \& Poggio, T. (2008). Dynamic population coding of category information in inferior temporal and prefrontal cortex. Journal of Neurophysiology, 100(3), 1407-1419.

Miller, J., \& Alderton, M. (2006). Backward response-level crosstalk in the psychological refractory period paradigm. Journal of Experimental Psychology. Human Perception and Performance, 32(1), 149-165.

Musslick, S., Saxe, A., Özcimder, K., Dey, B., Henselman, G., \& Cohen, J. D. (2017). Multitasking Capability Versus Learning Efficiency in Neural Network Architectures. CogSci, 829-834.

Navon, D., \& Miller, J. (2002). Queuing or sharing? A critical evaluation of the single-bottleneck notion. Cognitive Psychology, 44(3), 193-251.

Nichols, T. E. (2012). Multiple testing corrections, nonparametric methods, and random field theory. Neurolmage, 62(2), 811-815.

Olton, D. S., Wenk, G. L., Church, R. M., \& Meck, W. H. (1988). Attention and the frontal cortex as examined by simultaneous temporal processing. Neuropsychologia, 26(2), 307-318.

Paliwal, M., \& Kumar, U. A. (2009). Neural networks and statistical techniques: A review of applications. Expert Systems with Applications, 36(1), 2-17.

Pang, K. C., Yoder, R. M., \& Olton, D. S. (2001). Neurons in the lateral agranular frontal cortex have divided attention correlates in a simultaneous temporal processing task. Neuroscience, 103(3), 615-628.

Pashler, H. (1984). Processing stages in overlapping tasks: evidence for a central bottleneck. Journal of Experimental Psychology. Human Perception and Performance, 10(3), 358-377.

Pashler, H. (1990). Do response modality effects support multiprocessor models of divided attention? Journal of Experimental Psychology. Human Perception and Performance, 16(4), 826-842.

Pashler, H. (1994). Dual-task interference in simple tasks: data and theory. Psychological Bulletin, 116(2), 220-244.

Pashler, H., Jolicœur, P., Dell'Acqua, R., Crebolder, J., Goschke, T., De Jong, R., Meiran, N., Ivry, R. B., \& Hazeltine, E. (2000). Task switching and multitask performance. In S. Monsell (Ed.), Control of cognitive processes: Attention and performance XVIII , (pp (Vol. 779, pp. 275-423). The MIT Press, xvi.

Ratcliff, R., \& McKoon, G. (2008). The diffusion decision model: theory and data for two-choice decision tasks. Neural Computation, 20(4), 873-922.

Raymond, J. E., Shapiro, K. L., \& Arnell, K. M. (1992). Temporary suppression of visual processing in an RSVP task: an attentional blink? Journal of Experimental Psychology. Human Perception and Performance, 18(3), 849-860.

Redish, A. D., Jensen, S., Johnson, A., \& Kurth-Nelson, Z. (2007). Reconciling reinforcement learning models with behavioral extinction and renewal: implications for addiction, relapse, and problem gambling. Psychological Review, 114(3), 784-805.

Roelfsema, P. R., Lamme, V. A. F., \& Spekreijse, H. (2000). The implementation of visual routines. Vision Research, 40(10), 1385-1411.

Ruthruff, E., Hazeltine, E., \& Remington, R. W. (2006). What causes residual dual-task interference after practice? Psychological Research, 70(6), 494-503.

Ruthruff, E., Van Selst, M., Johnston, J. C., \& Remington, R. (2006). How does practice reduce dual-task interference: integration, automatization, or just stage-shortening? Psychological Research, 70(2), $125-142$.

Sacerdoti, E. D. (1974). Planning in a hierarchy of abstraction spaces. Artificial Intelligence, 5(2), $115-135$.

Sagiv, Y., Musslick, S., Niv, Y., \& Cohen, J. D. (2018). Efficiency of learning vs. processing: Towards a normative theory of multitasking. CogSci. http://mindmodeling.org/cogsci2018/papers/0200/0200.pdf

Salvucci, D. D., \& Taatgen, N. A. (2008). Threaded cognition: an integrated theory of concurrent multitasking. Psychological Review, 115(1), 101-130.

Schmitz, T. W., \& Duncan, J. (2018). Normalization and the Cholinergic Microcircuit: A Unified Basis for Attention. Trends in Cognitive Sciences, 22(5), 422-437. 
Schubert, T., \& Szameitat, A. J. (2003). Functional neuroanatomy of interference in overlapping dual tasks: an fMRI study. Brain Research. Cognitive Brain Research, 17(3), 733-746.

Serences, J. T., \& Saproo, S. (2012). Computational advances towards linking BOLD and behavior. Neuropsychologia, 50(4), 435-446.

Sergent, C., Baillet, S., \& Dehaene, S. (2005). Timing of the brain events underlying access to consciousness during the attentional blink. Nature Neuroscience, 8(10), 1391-1400.

Sigman, M., \& Dehaene, S. (2008). Brain mechanisms of serial and parallel processing during dual-task performance. The Journal of Neuroscience: The Official Journal of the Society for Neuroscience, 28(30), 7585-7598.

Smith, K. S., \& Graybiel, A. M. (2013). A dual operator view of habitual behavior reflecting cortical and striatal dynamics. Neuron, 79(2), 361-374.

Stelzel, C., Kraft, A., Brandt, S. A., \& Schubert, T. (2008). Dissociable neural effects of task order control and task set maintenance during dual-task processing. Journal of Cognitive Neuroscience, 20(4), 613-628.

Stelzel, C., Schumacher, E. H., Schubert, T., \& D'Esposito, M. (2006). The neural effect of stimulus-response modality compatibility on dual-task performance: an fMRI study. Psychological Research, 70(6), 514-525.

Sternberg, S. (1969). The discovery of processing stages: Extensions of Donders' method. Acta Psychologica, 30(0), 276-315.

Stokes, M. G., Kusunoki, M., Sigala, N., Nili, H., Gaffan, D., \& Duncan, J. (2013). Dynamic coding for cognitive control in prefrontal cortex. Neuron, 78(2), 364-375.

Strobach, T. (2019). The dual-task practice advantage: Empirical evidence and cognitive mechanisms. Psychonomic Bulletin \& Review. https://doi.org/10.3758/s13423-019-01619-4

Strobach, T., Frensch, P., Müller, H. J., \& Schubert, T. (2012a). Testing the limits of optimizing dual-task performance in younger and older adults. Frontiers in Human Neuroscience, 6, 39.

Strobach, T., Frensch, P., Müller, H., \& Schubert, T. (2012b). Age- and practice-related influences on dual-task costs and compensation mechanisms under optimal conditions of dual-task performance. Neuropsychology, Development, and Cognition. Section B, Aging, Neuropsychology and Cognition, 19(1-2), 222-247.

Strobach, T., Schütz, A., \& Schubert, T. (2015). On the importance of Task 1 and error performance measures in PRP dual-task studies. Frontiers in Psychology, 6, 403.

Sutton, R. S., \& Barto, A. G. (1998). Reinforcement Learning: An Introduction. MIT Press.

Szameitat, A. J., Lepsien, J., von Cramon, D. Y., Sterr, A., \& Schubert, T. (2006). Task-order coordination in dual-task performance and the lateral prefrontal cortex: an event-related fMRI study. Psychological Research, 70(6), 541-552.

Szameitat, A. J., Schubert, T., \& Müller, H. J. (2011). How to test for dual-task-specific effects in brain imaging studies--an evaluation of potential analysis methods. Neurolmage, 54(3), 1765-1773.

Szameitat, A. J., Schubert, T., Müller, K., \& Von Cramon, D. Y. (2002). Localization of executive functions in dual-task performance with fMRI. Journal of Cognitive Neuroscience, 14(8), 1184-1199.

Tamber-Rosenau, B. J., Dux, P. E., Tombu, M. N., Asplund, C. L., \& Marois, R. (2013). Amodal processing in human prefrontal cortex. The Journal of Neuroscience: The Official Journal of the Society for Neuroscience, 33(28), 11573-11587.

Telford, C. W. (1931). The refractory phase of voluntary and associative responses. Journal of Experimental Psychology. http://psycnet.apa.org/record/1931-04368-001

Tepper, J. M., \& Plenz, D. (n.d.). Striatal Cell Types and Their Interaction. Global Brain Function, 127.

Thoma, P., Koch, B., Heyder, K., Schwarz, M., \& Daum, I. (2008). Subcortical contributions to multitasking and response inhibition. Behavioural Brain Research, 194(2), 214-222.

Tombu, M., \& Jolicœur, P. (2003). A central capacity sharing model of dual-task performance. Journal of Experimental Psychology. Human Perception and Performance, 29(1), 3-18.

Ulrich, R., \& Miller, J. (2008). Response grouping in the psychological refractory period (PRP) paradigm: models and contamination effects. Cognitive Psychology, 57(2), 75-121.

Van Selst, M., Whitsell, S., \& Remington, R. (2003). Vanishing dual-task interference after practice: Has the bottleneck been eliminated or is it merely latent? Journal of. https://psycnet.apa.org/record/2003-00308-003?casa_token=kqWRHbYJviAAAAAA:AunjUbR-RkMSFfVmtzm 8870eP0UhdGkD9Bb7KrtnNL351QhRQQKRnLJ5rdOeVdYUZNEhFN93u5QZfDkDgwKQT6zA 
Watanabe, K., \& Funahashi, S. (2014). Neural mechanisms of dual-task interference and cognitive capacity limitation in the prefrontal cortex. Nature Neuroscience, 17(4), 601-611.

Watanabe, K., \& Funahashi, S. (2018). Toward an understanding of the neural mechanisms underlying dual-task performance: Contribution of comparative approaches using animal models. Neuroscience and Biobehavioral Reviews, 84, 12-28.

Welford, A. T. (1952). THE "PSYCHOLOGICAL REFRACTORY PERIOD" AND THE TIMING OF HIGH-SPEED PERFORMANCE-A REVIEW AND A THEORY. The British Journal of Psychology. General Section, 43(1), 2-19.

Welford, A. T. (1959). Evidence of a single-channel decision mechanism limiting performance in a serial reaction task. The Quarterly Journal of Experimental Psychology, 11(4), 193-210.

Welford, A. T. (1967). Single-channel operation in the brain. Acta Psychologica, 27, 5-22.

Woolgar, A., Hampshire, A., Thompson, R., \& Duncan, J. (2011). Adaptive coding of task-relevant information in human frontoparietal cortex. The Journal of Neuroscience: The Official Journal of the Society for Neuroscience, 31(41), 14592-14599.

Woolgar, A., Jackson, J., \& Duncan, J. (2016). Coding of Visual, Auditory, Rule, and Response Information in the Brain: 10 Years of Multivoxel Pattern Analysis. Journal of Cognitive Neuroscience, 28(10), 1433-1454.

Yildiz, A., \& Beste, C. (2015). Parallel and serial processing in dual-tasking differentially involves mechanisms in the striatum and the lateral prefrontal cortex. Brain Structure \& Function, 220(6), 3131-3142.

Yin, H. H., \& Knowlton, B. J. (2006). The role of the basal ganglia in habit formation. Nature Reviews. Neuroscience, 7(6), 464-476.

Yosinski, J., Clune, J., Bengio, Y., \& Lipson, H. (2014). How transferable are features in deep neural networks? In Z. Ghahramani, M. Welling, C. Cortes, N. D. Lawrence, \& K. Q. Weinberger (Eds.), Advances in Neural Information Processing Systems 27 (pp. 3320-3328). Curran Associates, Inc.

Zylberberg, A., Fernández Slezak, D., Roelfsema, P. R., Dehaene, S., \& Sigman, M. (2010). The brain's router: a cortical network model of serial processing in the primate brain. PLoS Computational Biology, 6(4), e1000765. 